\title{
Densities Probed by Coronal Type III Radio Burst Imaging
}

\author{
Patrick I. McCauley ${ }^{1}$ (D) Iver H. Cairns ${ }^{1}$ (D) John Morgan $^{2}$

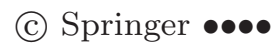

\begin{abstract}
We present coronal density profiles derived from low-frequency (80-240 MHz) imaging of three type III solar radio bursts observed at the limb by the Murchison Widefield Array (MWA). Each event is associated with a white light streamer at larger heights and is plausibly associated with thin extreme ultraviolet rays at lower heights. Assuming harmonic plasma emission, we find average electron densities of $1.8 \times 10^{8} \mathrm{~cm}^{-3}$ down to $0.20 \times 10^{8} \mathrm{~cm}^{-3}$ at heights of 1.3 to $1.9 \mathrm{R}_{\odot}$. These values represent $\sim 2.4-5.4 \times$ enhancements over canonical background levels and are comparable to the highest streamer densities obtained from data at other wavelengths. Assuming fundamental emission instead would increase the densities by a factor of 4 . High densities inferred from type III source heights can be explained by assuming that the exciting electron beams travel along overdense fibers or by radio propagation effects that may cause a source to appear at a larger height than the true emission site. We review the arguments for both scenarios in light of recent results. We compare the extent of the quiescent corona to model predictions to estimate the impact of propagation effects, which we conclude can only partially explain the apparent density enhancements. Finally, we use the time- and frequency-varying source positions to estimate electron beam speeds of between 0.24 and $0.60 \mathrm{c}$.
\end{abstract}

Keywords: Radio Bursts, Type III; Radio Bursts, Meter-Wavelengths and Longer; Corona, Radio Emission; Corona, Structures; Density; Streamers; Spectroscopic Imaging

\section{Introduction}

Type III solar radio bursts are caused by semi-relativistic electrons streaming through and perturbing the ambient coronal or interplanetary plasma. A recent review is given by

\footnotetext{
$凶$ P. I. McCauley

patrick.mccauley@sydney.edu.au

$\bigotimes$ I. H. Cairns

iver.cairns@sydney.edu.au

$\bowtie$ J. Morgan

john.morgan@curtin.edu.au

1 School of Physics, University of Sydney, Sydney, NSW 2006, Australia

2 International Centre for Radio Astronomy Research, Curtin University, Perth, WA 6845, Australia
} 
Reid and Ratcliffe (2014). The dominant theory, proposed by Ginzburg and Zhelezniakov (1958), invokes a two-step process beginning with the stimulation of Langmuir waves (plasma oscillations) in the background plasma by an electron beam. A small fraction of the Langmuir wave energy is then converted into electromagnetic radiation at either the local electron plasma frequency $\left(f_{p}\right)$ or its harmonic $\left(2 f_{p}\right.$; see reviews by Robinson and Cairns 2000; Melrose 2009). The emission frequency depends mainly on the ambient electron density $\left(n_{e}\right)$ because $f_{p} \propto \sqrt{n_{e}}$. This relationship produces the defining feature of type III bursts, a rapid drift from high to low frequencies as the exciter beam travels away from the Sun through decreasing densities (Wild and McCready, 1950).

The rate at which the emission frequency drifts $(d f / d t)$ is therefore related to the electron beam speed, which can be obtained in the radial direction by assuming a density model $n_{e}(r)$. Many authors have employed this technique for various events with various models, generally finding modest fractions of light speed (0.1-0.4 c; e.g. Alvarez and Haddock 1973; Aschwanden et al. 1995; Mann et al. 1999; Meléndez et al. 1999; Krupar et al. 2015; Kishore et al. 2017). Alternatively, the coronal and/or interplanetary density gradient can be inferred by instead assuming a beam speed (e.g. Fainberg and Stone 1971; Leblanc, Dulk, and Bougeret 1998) or by simply assuming that the beam speed is constant (Cairns et al., 2009). While these methods can yield robust estimates for the density gradient, they cannot be converted into an explicit density structure $n_{e}(r)$ without normalizing the gradient to a specific value at a specific heliocentric distance. This normalization has typically been done using estimates from white light polarized brightness data close to the Sun, in situ data in the interplanetary medium, or the observed height of type III burst sources at various frequencies.

Densities inferred from type III source heights, particularly at lower frequencies, have frequently conflicted with those obtained from other methods. The earliest spatial measurements found larger source heights than would be expected from fundamental plasma emission, implying density enhancements of an order of magnitude or more (Wild, Sheridan, and Neylan, 1959). This finding was confirmed by subsequent investigations (e.g. Morimoto 1964; Malitson and Erickson 1966), and along with other arguments, led many authors to two conclusions: First, that harmonic $\left(2 f_{p}\right)$ emission likely dominates (e.g. Fainberg and Stone 1971; Mercier and Rosenberg 1974; Stewart 1976). This brings the corresponding densities down by a factor of 4, then implying only a moderate enhancement over densities inferred from white light data. (Counterarguments for the prevalence of fundamental emission will be referenced in Section 3.1.) Second, that the electron beams preferentially traverse overdense flux tubes (e.g. Bougeret, King, and Schwenn 1984), a conclusion bolstered by spatial correlations between several type III bursts and white light streamers (e.g. Trottet et al. 1982; Kundu and Stone 1984; Gopalswamy, Kundu, and Szabo 1987; Mugundhan et al. 2018).

The overdense hypothesis has been challenged by evidence that the large source heights can instead be explained by propagation effects. If type III emission is produced in thin, high-density structures, then it can escape relatively unperturbed through its comparatively rarefied surroundings. However, if the emission is produced in an environment near the associated plasma level (i.e. with an average $n_{e}$ corresponding to the radio waves' equivalent $f_{p}$ ), then refraction and scattering by density inhomogeneities may substantially shift an observed source from its true origin (e.g. Leblanc 1973; Riddle 1974; Bougeret and Steinberg 1977). Duncan (1979) introduced the term ducting in this context, which refers to emission being guided to larger heights within a low-density structure though successive reflections against the high-density "walls" of the duct. This concept was generalized 
for a more realistic corona by Robinson (1983), who showed that random scattering of radio waves by thin, overdense fibers has the same net effect of elevating an observed source radially above its emission site. Additional details on this topic, along with coronal refraction, will be given in Section 3.3.

Many authors came to favor propagation effects instead of the overdense structure interpretation for a few reasons. Despite the aforementioned case studies, type IIIs did not appear to be statistically associated with regions of high average density in the corona (Leblanc, Kuiper, and Hansen, 1974; Leblanc and de La Noe, 1977) or in the solar wind (Steinberg et al., 1984). Interplanetary (kHz-range) type III source regions are also so large as to demand angular broadening by propagation effects (e.g. Steinberg, Hoang, and Dulk 1985; Lecacheux et al. 1989). Invoking propagation effects can also be used to explain apparent spatial differences between fundamental and harmonic sources (e.g. Stewart 1972; Kontar et al. 2017), along with large offsets between radio sources on the disk and their likely electron acceleration sites (e.g. Bisoi et al. 2018). These arguments are reviewed by Dulk (2000), and further discussion with additional recent references will be presented in Sections 3.3 and 4.

Both the interpretation of electron beams moving along overdense structures and of radio propagation effects elevating burst sources rely on the presence of thin, high-density fibers. Either the electron beams are traveling within these structures or the type III emission is being scattered by them. In this paper, we will suggest that propagation effects are important but cannot entirely explain the density enhancements for our events. Section 2 describes our observations: 2.1 outlines our data reduction, 2.2 details our event selection criteria, and 2.3 describes the multi-wavelength context for the selected type III bursts. Section 3 describes our analysis and results: 3.1 infers densities from type III source heights, 3.2 estimates electron beam speeds from imaging data, and 3.3 examines propagation effects by comparing the extent of the quiescent corona to model predictions. In Section 4, we discuss the implications of our results, along with other recent developments, on the debate between the overdense and propagation effects hypotheses. Finally, our conclusions are summarized in Section 5.

\section{Observations}

\subsection{Murchison Widefield Array (MWA)}

The MWA is a low-frequency radio interferometer in Western Australia with an instantaneous bandwidth of $30.72 \mathrm{MHz}$ that can be flexibly distributed from 80 to $300 \mathrm{MHz}$ (Tingay et al., 2013). Our data were recorded with a $0.5 \mathrm{~s}$ time cadence and a $40 \mathrm{kHz}$ spectral resolution, which we average over 12 separate $2.56 \mathrm{MHz}$ bandwidths centered at $80,89,98,108,120,132,145,161,179,196,217$, and $240 \mathrm{MHz}$. We use the same data processing scheme as McCauley et al. (2017), and what follows is a brief summary thereof.

Visibilities were generated with the standard MWA correlator (Ord et al., 2015) and the cotter software (Offringa, van de Gronde, and Roerdink, 2012; Offringa et al., 2015). Observations of bright and well-modelled calibrator sources were used to obtain solutions for the complex antenna gains (Hurley-Walker et al., 2014), which were improved by imaging the calibrator and iteratively self-calibrating from there (Hurley-Walker et al., 2017). WSClean (Offringa et al., 2014) was used to perform the imaging with a Briggs -2 weighting (Briggs, 1995) to maximize spatial resolution and minimize point spread function (PSF) sidelobes. The primary beam model of Sutinjo et al. (2015) was used 


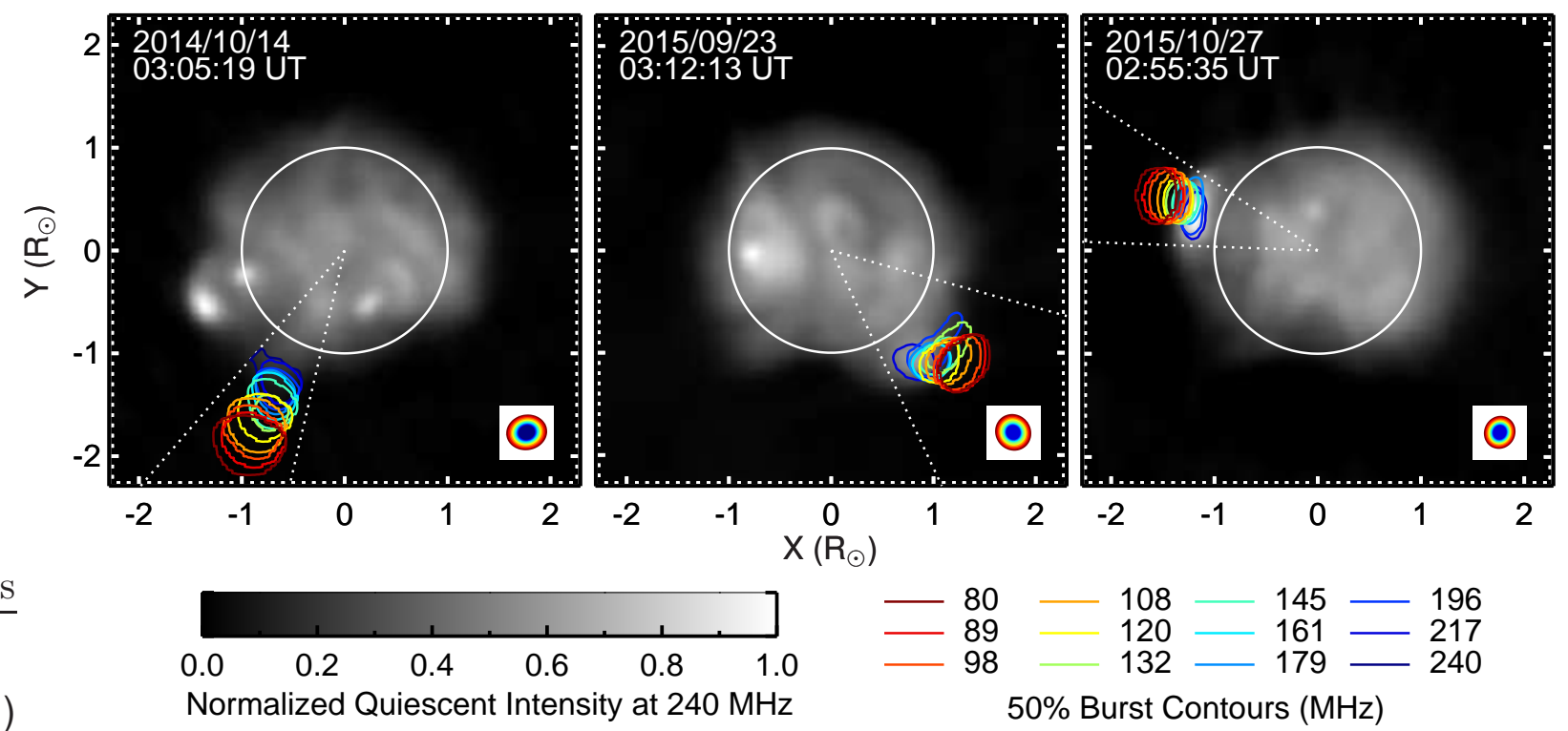

Figure 1. MWA type III burst contours at $50 \%$ of the peak intensity for each channel overlaid on 240 $\mathrm{MHz}$ images of the quiescent corona. The solid circle represents the optical disk, and dotted lines bound the region included in the dynamic spectra (Figs. 2 \& 3). Colored ellipses in the lower-right corners show the synthesized beam sizes for each channel.
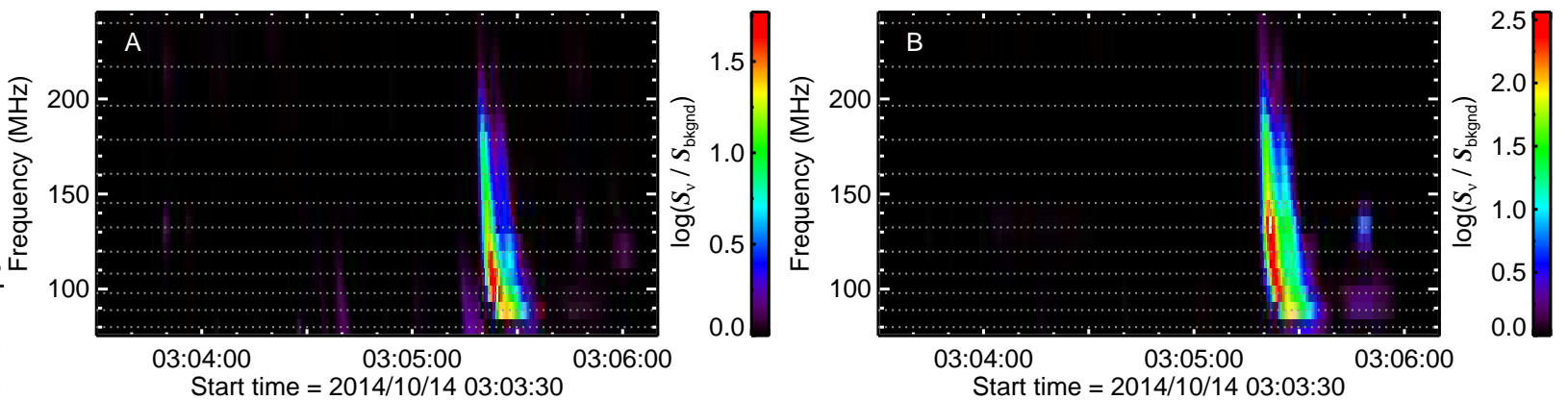

Figure 2. Dynamic spectra constructed from image intensities for the type III burst near 03:05:20 UT on 2014-10-14. Panel A includes the full FOV, while B includes only the segment bounded by the dotted lines in Fig. 1. Dotted horizontal lines show the locations of the 12 channels, each having a spectral width of $2.56 \mathrm{MHz}$. Intensities have been divided by the background level and plotted a logarithmic scale.

to produce Stokes I images from the instrumental polarizations, and the SolarSoftWare (SSW ${ }^{1}$, Freeland and Handy 1998) routine mwa_prep (McCauley et al., 2017) was used to translate the images onto solar coordinates. Flux calibration was achieved by comparison with thermal bremsstrahlung and gyroresonance emission predictions from FORWARD ${ }^{2}$ (Gibson et al., 2016) based on the Magnetohydrodynamic Algorithm outside a Sphere model (MAS ${ }^{3}$; Lionello, Linker, and Mikić 2009).

\subsection{Event Selection}

These data are part of an imaging survey of many type III bursts observed by the MWA during 45 separate observing periods in 2014 and 2015. McCauley et al. (2017) performed a case study of an event that exhibits unusual source motion, and future work will present

\footnotetext{
${ }^{1} \mathrm{SSW}:$ https://www.Imsal.com/solarsoft/

${ }^{2}$ FORWARD: https://www2.hao.ucar.edu/modeling/FORWARD-home

${ }^{3}$ MAS: http://www.predsci.com/hmi/data_access.php
} 

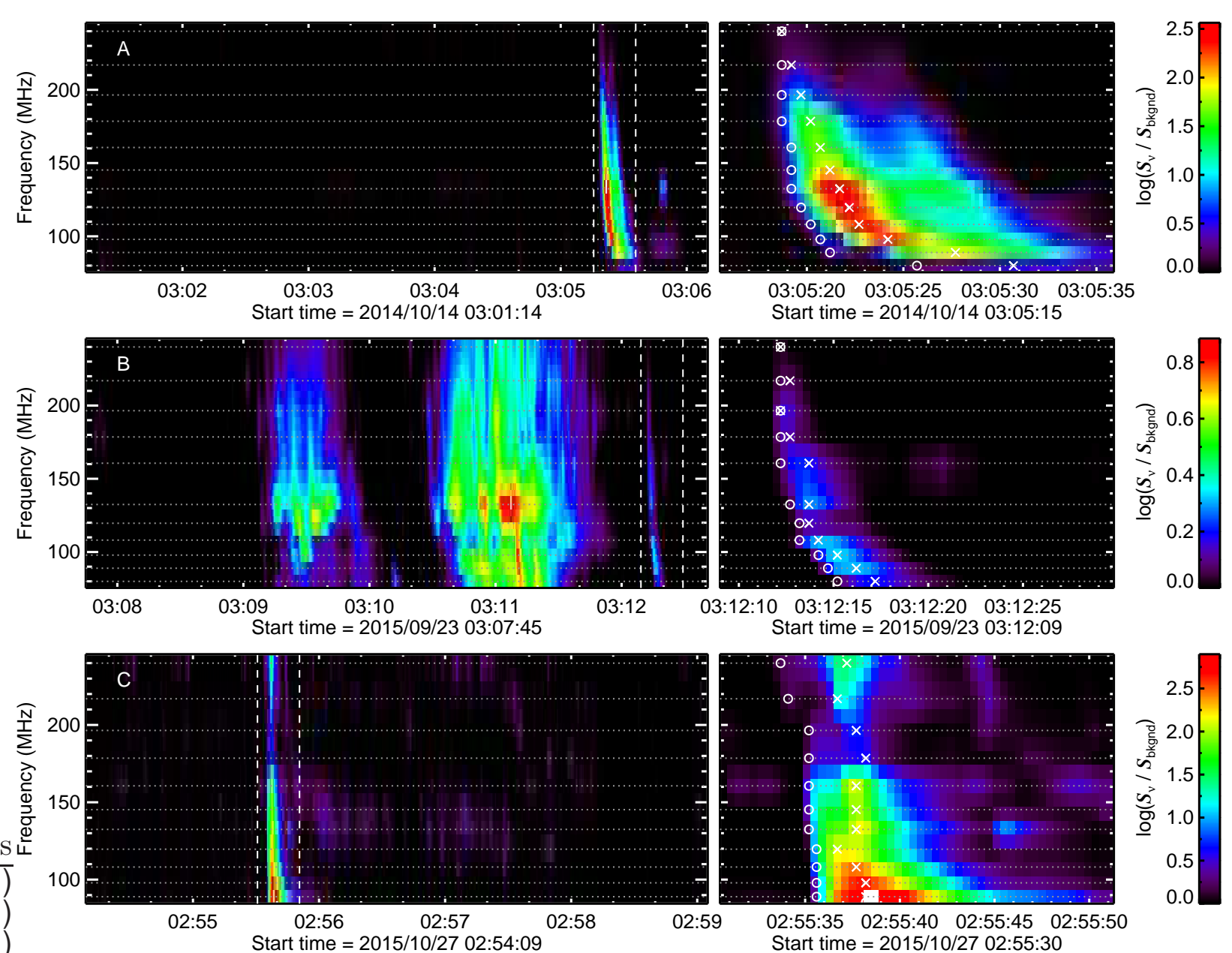

Figure 3. Dynamic spectra constructed from partial image intensities, including only the FOV segment bounded by the dotted lines in Fig. 1. The left column shows the full 5-min observation intervals, while the right column shows 20 -sec periods surrounding the selected type III bursts. Circles and crosses denote the onset and peak burst times for each channel.

statistical analyses. Burst periods during MWA observing runs were identified using the daily National Oceanic and Atmospheric Administration (NOAA) solar event reports ${ }^{4}$ based on observations from the Learmonth (Guidice et al., 1981; Kennewell and Steward, 2003) and Culgoora (Prestage et al., 1994) solar radio spectrographs, which overlap with the MWA's frequency range at the low and high ends, respectively.

Three events were selected from the full sample based on the following criteria. First, the burst sites needed to be located at the radio limb with roughly radial progressions across frequency channels. Limb events minimize projection effects, allowing us to reasonably approximate the projected distance from Sun-center as the actual radial height. Second, to eliminate potential confusion between multiple events and to maximize spectral coverage, the bursts needed to be sufficiently isolated in time and frequency, with a coherent drift from high to low frequencies across the full MWA bandwidth. Third, the source regions needed to be relatively uncomplicated ellipses with little-to-no intrinsic motion of the sort described by McCauley et al. (2017). This again minimizes projection effects and ensures that we follow a single beam trajectory for each event.

\footnotetext{
${ }^{4}$ NOAA event reports: http://www.swpc.noaa.gov/products/solar-and-geophysical-event-reports
} 
Figure 1 shows the burst contours for each channel overlaid on quiescent background images at $240 \mathrm{MHz}$. Each of the three events occurred on a different day, and we refer to them by the UTC date on which they occurred. Figure 2 shows dynamic spectra for the 2014-10-14 event, with the left panel covering the full Sun and the right panel including only the region demarcated by the dotted lines in Figure 1. The partial Sun spectrum excludes a neighboring region that is active over the same period, allowing the type III frequency structure to be more easily followed. This approach is similar to that of Mohan and Oberoi (2017), who discuss the utility of spatially resolved dynamic spectra. Figure 3 shows the masked spectra for all three events.

\subsection{Context}

In this section, we briefly describe the context for each of the radio bursts with respect to observations at other wavelengths and associated phenomena. Figure 4 overlays the burst contours from Figure 1 onto contemporaneous extreme ultraviolet (EUV) and white light data. The white light images were produced by the Large Angle and Spectrometric C2 Coronagraph (LASCO C2; Brueckner et al. 1995) onboard the Solar and Heliospheric Observatory (SOHO; Domingo, Fleck, and Poland 1995). C2 has an observing cadence of 20 min, and Figure 4 includes the nearest images in time to our radio bursts.

The EUV data come from the Atmospheric Imaging Assembly (AIA; Lemen et al. 2012) onboard the Solar Dynamics Observatory (SDO; Pesnell, Thompson, and Chamberlin 2012). We use the $171 \AA$ AIA channel, which is dominated by Fe IX emission produced by plasma at around $0.63 \mathrm{MK}$, because it most clearly delineates the fine magnetic structures along which type III beams are expected to travel. To further accentuate off-limb features, we apply a radial filter using the SSW routine aia_rfilter (Masson et al., 2014). Note that the apparent brightness of a given pixel in a radial filter image corresponds to its true intensity relative only to pixels of the same radial height (i.e. equally bright structures at different heights do not have the same physical intensity). AIA has an observing cadence of $12 \mathrm{~s}$, and Figure 4 uses 10-min (50-image) averages that cover the periods during and immediately after the radio bursts. This time window is used because a potential EUV signature associated with a type III burst will propagate at a much lower speed than the burst-driving electron beam and will likely be most apparent in the minutes following the burst (e.g. McCauley et al. 2017; Cairns et al. 2018).

In all cases, the radio bursts appear to be aligned with dense structures visible to AIA at lower heights and to LASCO C2 at larger heights. The latter case is obvious, with each set of burst contours situated just below bright white light streamers. Cyan arrows in the right panels of Figure 4 identify the associated EUV structures, each of which exhibits a mild brightening and/or outflow during or immediately after the corresponding radio burst. This activity may be indicative of weak EUV jets, which are frequently associated with type III bursts (e.g. Chen et al. 2013; Innes et al. 2016; McCauley et al. 2017; Cairns et al. 2018), but robust outflows are not observed here. The alignment between the EUV and radio burst structure is particularly striking for the 2015-09-23 event in that both appear to follow roughly the same non-radial arc. A correspondence between EUV rays and type III bursts was previously reported by Pick et al. (2009).

Type III bursts are commonly, but not always, associated with X-ray flares (e.g. Benz et al. 2005; Benz, Brajša, and Magdalenić 2007; Cairns et al. 2018) and occasionally with Coronal Mass Ejections (CMEs; e.g. Cane, Erickson, and Prestage 2002; Cliver and Ling 2009). Our 2014-10-14 event is not associated with either, but the other two are. On 201509-23, a weak B-class flare occurred just to the north of our radio sources from active region 

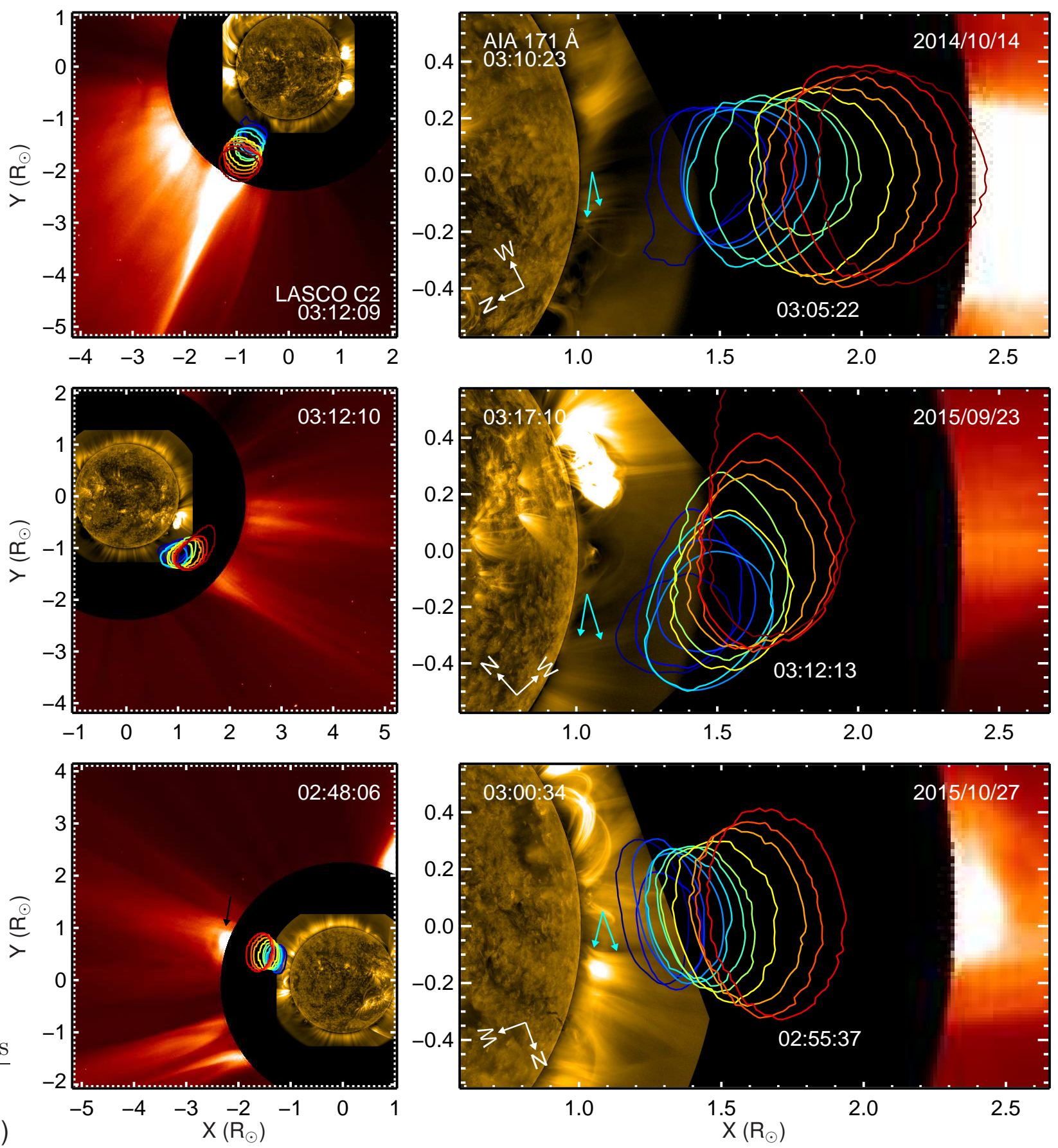

Figure 4. Overlays of the $50 \%$ burst contours onto AIA $171 \AA$ and LASCO C2 images. Contour colors are for spectral channels from $80-240 \mathrm{MHz}$ as in Fig. 1. UTC observation times are shown for LASCO in the left panel, for AIA in the upper-left of the right panel, and for MWA in the middle of the right panel. The MWA times reflect the average peak time across frequency channels (see Fig. 3). The AIA images are 10-min (50-image) averages processed with a radial filter to accentuate off-limb features; times reflect the middle of these 10-min windows, which begin at the burst onsets and cover the subsequent periods over which associated EUV signatures would be expected. Images are rotated in the right column such that the burst progression is roughly horizontal, which helps illustrate the extent to which each event progresses radially. Cyan arrows point to the EUV structures that exhibit activity during or just after the radio bursts. The black arrow in the lower-left panel points to a CME that originated behind the limb and passed the C2 occulting disk around 20-min prior to the type III burst. 


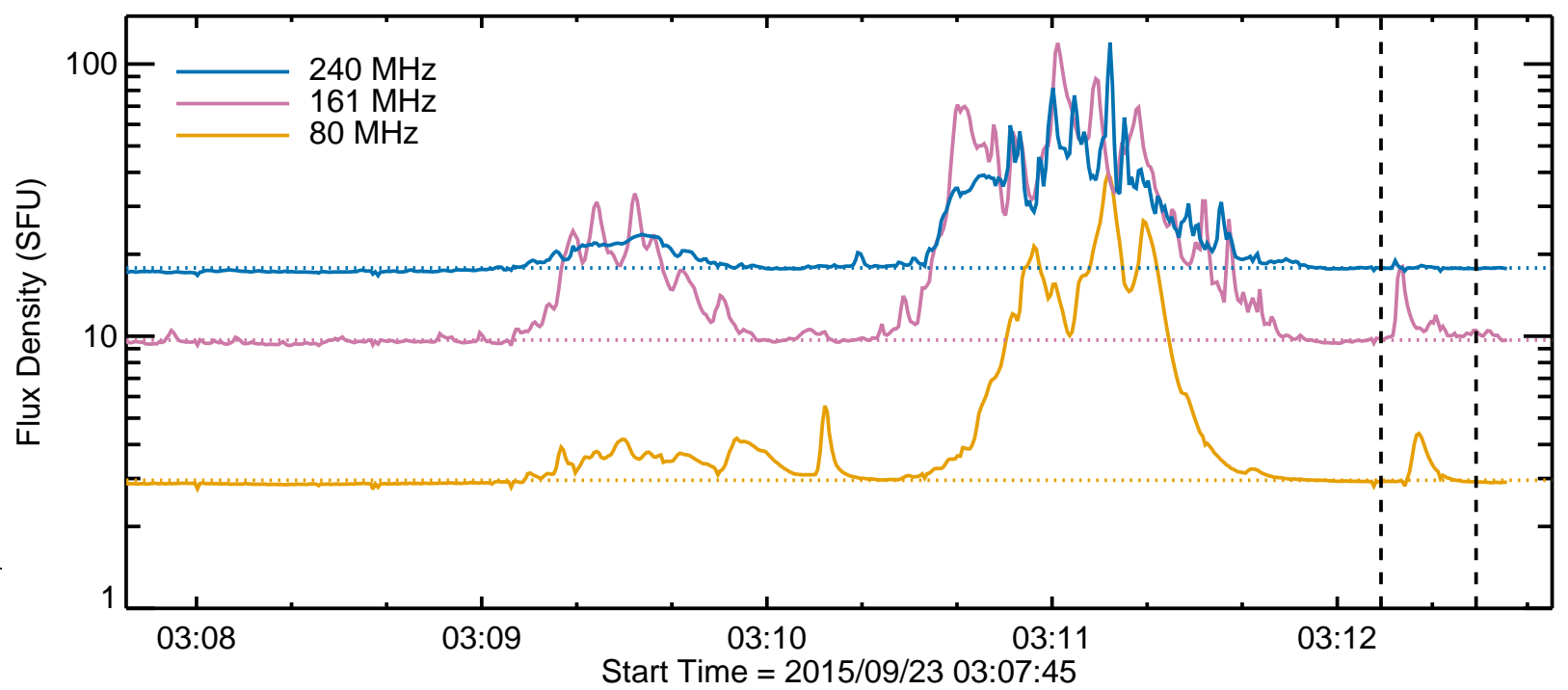

Figure 5. Light curves for the 2015-09-23 observation, shown to illustrate the background level determination. Backgrounds (dotted lines) are obtained by taking the median intensity, excluding points 2 standard deviations above that, and iterating until no more points are excluded. The dashed lines mark the burst period from the right column of Fig. 3 .

12415. The flare peaked around 3:11 UT, which corresponds to a period of relatively intense coherent radio emission that precedes the weaker burst of interest here (see Figure 3). Given the radio source positions and associated EUV structure, we do not believe the flare site to be the source of accelerated electrons for our event, though the flare may have been responsible for stimulating further reconnection to the south.

On 2015-10-27, a CME was ongoing at the time of the radio burst, and its leading edge, indicated by the black arrow in the lower left panel of Figure 4, can be seen just above the C2 occulting disk. Inspection of images from the Extreme Ultraviolet Imager (EUVI; Howard et al. 2008) onboard the STEREO-A spacecraft shows that the CME originated from a large active region close to the east limb but occulted by the disk from AIA's perspective. The CME was launched well before our type III burst, but the region that produced it was very active over this period and is likely connected to the activity visible to AIA immediately after the radio burst along the structure indicated by the cyan arrows in the lower right panel of Figure 4. So while we do not think the CME was directly involved in triggering the radio burst, it may have impacted the medium through which the type III electron beam would later propagate, which is relevant to a hypothesis proposed by Morosan et al. (2014) that will be discussed in Section 4.

\section{Analysis and Results}

\subsection{Density Profiles}

Standard plasma emission theory expects type III radiation at either the ambient electron plasma frequency $\left(f_{\mathrm{p}}\right)$ or its harmonic $\left(2 f_{\mathrm{p}}\right)$. The emission frequency $f$ is related to electron density $\left(n_{e}\right)$ in the following way (in cgs units):

$$
f=\mathrm{N} f_{\mathrm{p}}=\mathrm{N} \sqrt{\frac{e^{2} n_{e}}{\pi m_{e}}} \Rightarrow n_{e}=\pi m_{e}\left(\frac{f}{\mathrm{Ne}}\right)^{2}
$$


where $e$ is the electron charge, $m_{e}$ is the electron mass, and $\mathrm{N}$ is either 1 (fundamental) or 2 (harmonic). For frequencies in $\mathrm{Hz}$ and densities in $\mathrm{cm}^{-3}, n_{e} \approx 1.24 \times 10^{-8} f^{2}$ for fundamental and $3.10 \times 10^{-9} f^{2}$ for harmonic emission.

Density can thus be easily extracted given the emission mode and location. Unfortunately, neither property is entirely straightforward. Harmonic emission is often favored in the corona because being produced above the ambient $f_{p}$ makes it less likely to absorbed (Bastian, Benz, and Gary, 1998) and because type IIIs tend to be more weakly circularly polarized than expected for fundamental emission (Dulk and Suzuki, 1980). Harmonic emission also implies lower densities by a factor of 4 , which are easier to reconcile with the large heights often observed (see Section 1). However, fundamental-harmonic pairs can be observed near our frequency range (e.g. Kontar et al. 2017), fundamental emission is expected to contribute significantly to interplanetary type III burst spectra (e.g. Robinson and Cairns 1998), and fundamental emission is thought to be the more efficient process from a theoretical perspective (e.g. Li and Cairns 2013, 2014). As described in Section 1, a source's apparent height may also be augmented by propagation effects, which we will consider in Section 3.3.

We measure source heights at the onset of burst emission, which we define as when the total intensity reaches $1.3 \times$ the background level. Background levels are determined for each frequency by taking the median intensity, excluding points 2 standard deviations above that, and iterating until no more points are excluded. Figure 5 shows the result of this baseline procedure for three frequencies from the 2015-09-23 event, which is shown because it exhibits the most complicated dynamic spectrum. Onset times are represented by circles in Figure 3, and centroids are obtained at these times from 2-dimensional (2D) Gaussian fits. As mentioned in Section 2.2, these events were chosen because they appear at the radio limb and thus the 2D plane-of-sky positions can reasonably approximate the physical altitude. Geometrically, these heights are lower limits to the true radial height, but propagation effects that increase apparent height are likely to be more important than the projection angle (see Section 3.3).

Figure 6 plots height versus density for both the fundamental and harmonic assumptions. Two sets of height uncertainties are shown for the average density profiles. The smaller, dark gray error bars reflect the $1 \sigma$ position variability over the full burst durations, and the larger, light gray bars reflect the full width at half maximum (FWHM) of the synethesized beam major axes. Note that if the source is dominated by a single compact component, which would be a reasonable assumption here, then the FWHM resolution uncertainty can be reduced by a factor inversely proportional to the signal-to-noise ratio (SNR) (Lonsdale et al., 2018; Reid et al., 1988). Given our high SNRs, which average $217 \sigma$ at the burst onsets, this "spot mapping" approach typically results in sub-arcsecond position uncertainties on the apparent source location. However, spatial shifts may be introduced by changes in the ionosphere between the solar and calibration observation times, and more importantly, an apparent source may differ significantly from its actual emission site due to propagation effects (i.e. refraction and scattering). For these reasons, we opt to show the more conservative uncertainties outlined above.

For comparison, Figure 6 includes radial density models from Saito, Poland, and Munro (1977), Newkirk (1961), and Goryaev et al. (2014). The Saito, Poland, and Munro profile refers to the equatorial background near solar minimum based on white light polarized brightness data, while the Newkirk curve is based on similar data near solar maximum and implies the largest densities among "standard" background models. The Goryaev et al. 


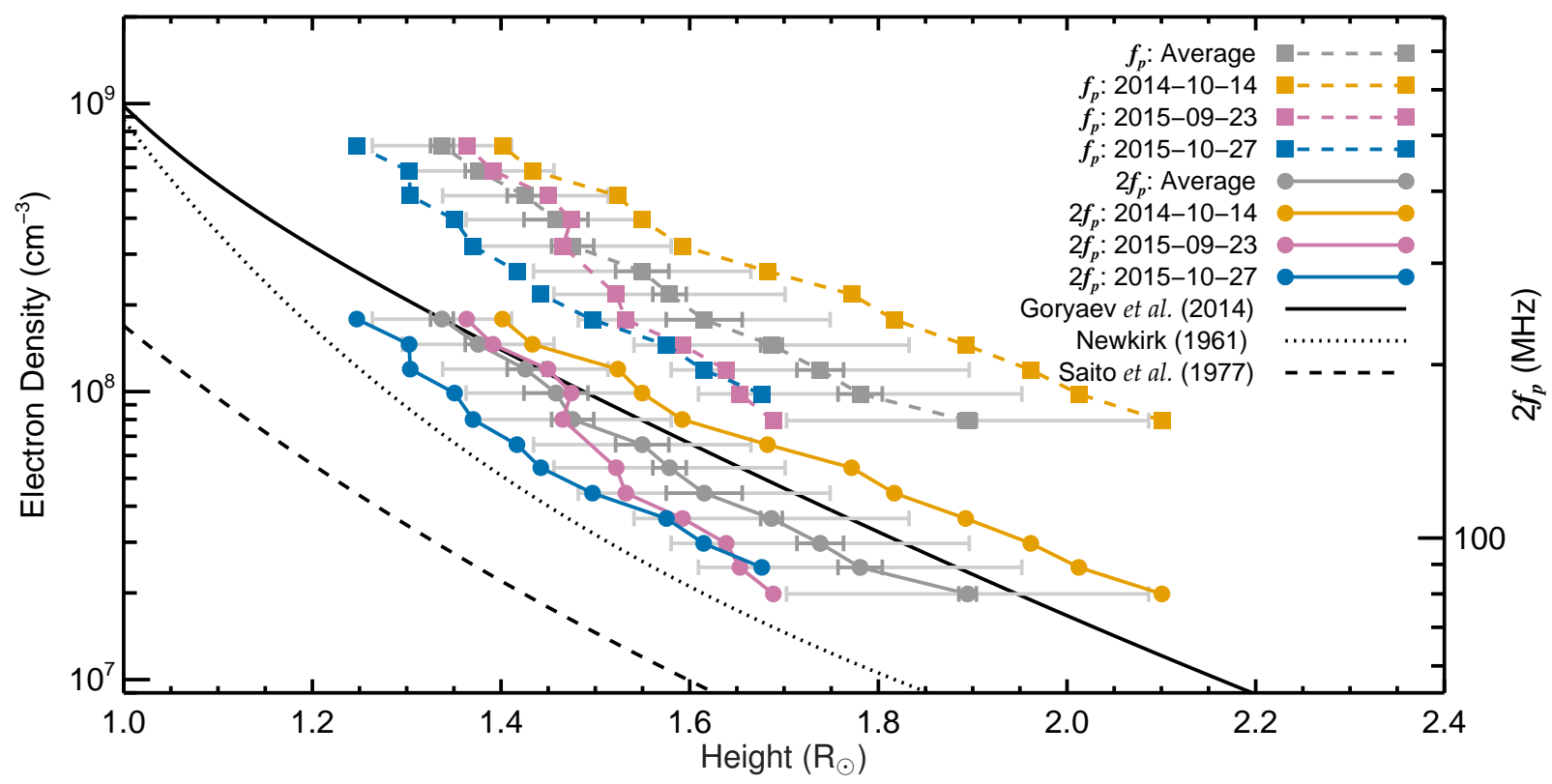

Figure 6. Densities inferred from the type III source positions assuming fundamental $\left(f_{p}\right.$; dashed) or harmonic $\left(2 f_{p}\right.$; solid) emission. Background coronal models based on white light data near solar minimum (Saito, Poland, and Munro, 1977) and maximum (Newkirk, 1961) are shown for comparison, along with a recent streamer model based on EUV data (Goryaev et al., 2014). Only the average uncertainties are shown for clarity; the dark gray bars represent the $1 \sigma$ centroid variability over the full burst, and the light gray bars represent the major axes of the synthesized beams.

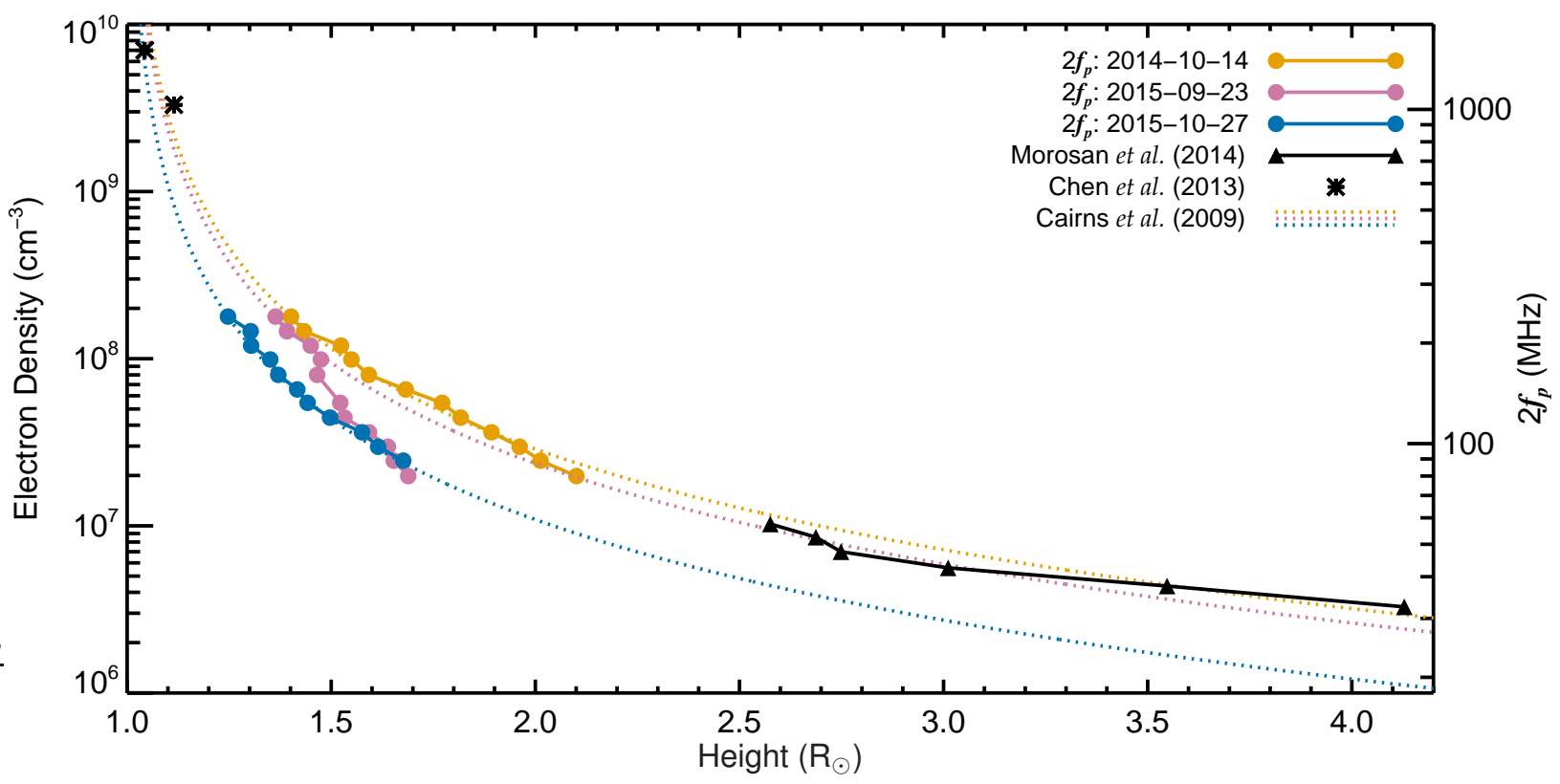

Figure 7. Densities assuming harmonic emission compared to recent type III results at higher (Chen et al., 2013) and lower (Morosan et al., 2014) frequencies. The dotted lines apply the $n_{e}(r)=C(r-1)^{-2}$ profile detailed by Cairns et al. (2009), where the constant $C$ has been normalized to the density implied by our $240 \mathrm{MHz}$ source positions. 
model instead refers to a dense streamer and is based on a novel technique using widefield EUV imaging. This profile is somewhat elevated above streamer densities inferred from contemporary white light (e.g. Gibson et al. 1999) and spectroscopic (e.g. Parenti et al. 2000; Spadaro et al. 2007) measurements at similar heights, though some earlier white light studies found comparably large streamer densities (e.g. Saito and Owaki 1967). For additional coronal density profiles, see also Allen (1947); Koutchmy (1994); Guhathakurta, Holzer, and MacQueen (1996); Mann et al. (1999); Mercier and Chambe (2015); Wang et al. (2017) and references therein.

From Figure 6, we see that the type III densities assuming fundamental emission are an average of 3-4× higher than the EUV streamer model. These values may be unreasonably large, meaning either that the fundamental emission hypothesis is not viable here or that fundamental emission originating from a lower altitude was observed a larger height due to propagation effects (see Section 3.3). Assuming harmonic emission, the 2014-10-14 burst implies electron densities of $1.8 \times 10^{8} \mathrm{~cm}^{-3}(240 \mathrm{MHz})$ at $1.40 \mathrm{R}_{\odot}$ down to $0.20 \times 10^{8} \mathrm{~cm}^{-3}$ $(80 \mathrm{MHz})$ at $2.10 \mathrm{R}_{\odot}$. This represents a moderate $(\sim 1.4 \times)$ enhancement over the Goryaev et al. streamer model or a significant $(\sim 4.1 \times)$ enhancement over the Newkirk background. The other two events fall between the EUV streamer and solar maximum background models, with the 2015-10-27 source heights implying densities of $1.8 \times 10^{8} \mathrm{~cm}^{-3}(240 \mathrm{MHz})$ at $1.25 \mathrm{R}_{\odot}$ down to $0.20 \times 10^{8} \mathrm{~cm}^{-3}(80 \mathrm{MHz})$ at $1.68 \mathrm{R}_{\odot}$. Note that the $2015-09-23$ burst implies an unusually steep density gradient that is not consistent with standard radial density models, perhaps because that event deviates significantly from the radial direction (see Figure 4).

Figure 7 shows how our results compare to densities inferred from recent type III imaging at higher and lower frequencies, all assuming harmonic emission. The high-frequency (1.0$1.5 \mathrm{GHz}$ ) results come from Chen et al. (2013), who used the Very Large Array (VLA) to find densities around an order of magnitude above the background. The low-frequency (30-60 MHz) points were obtained using the Low Frequency Array (LOFAR) by Morosan et al. (2014), who also found large enhancements. We plot data from their "Burst 2" (see Figures $3 \& 4$ ) because it began beyond our average radio limb height at $80 \mathrm{MHz}$. Their other two events exhibit $60 \mathrm{MHz}$ emission near the optical limb, which may indicate that the 2D plane-of-sky positions significantly underestimate the true altitudes (i.e. those electron beams may have been inclined toward the observer).

Figure 7 also includes density curves of the form $n_{e}(r)=C(r-1)^{-2}$, where $r$ is in solar radii and $C$ is normalized to match the densities implied by our $240 \mathrm{MHz}$ source heights. This model was introduced by Cairns et al. (2009) based on type III frequency drift rates over $40-180 \mathrm{MHz}$ and was subsequently validated over a larger frequency range by Lobzin et al. (2010). The Cairns et al. model is somewhat steeper than others over the MWA's height range $\left(\sim 1.25-2.10 \mathrm{R}_{\odot}\right)$ but becomes more gently-sloping at larger heights, effectively bridging the corona to solar wind transition. From Figure 7, we see that this model is a good fit to the 2014-10-14 and 2015-10-27 data. The 2015-09-23 event is not wellfit by this or any other standard model, which may be attributed to its aforementioned non-radial structure. Extending these gradients to larger heights matches the LOFAR data fairly well and likewise with the VLA data at lower heights, which come from higher frequencies than have been examined with this model previously.

\subsection{Electron Beam Kinematics}

Type III beams speeds are known primarily from frequency drift rates $(d f / d t)$ observed in dynamic spectra. Assuming either fundamental or harmonic emission, a given burst 


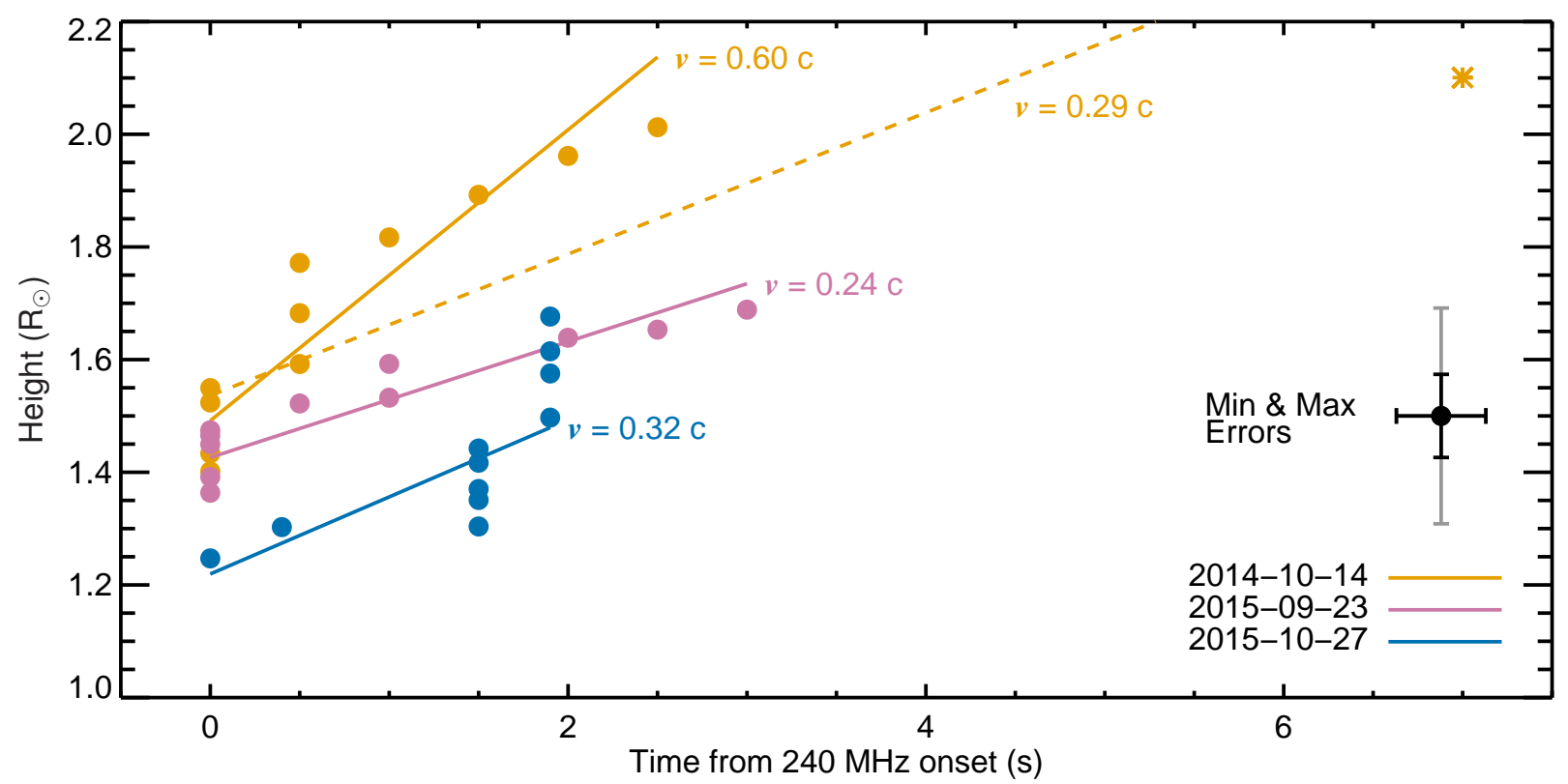

Figure 8. Exciter speed estimates from the time- and frequency-varying source positions. The dashed orange line includes the high time outlier (orange asterisk). The uncertainties shown in the lower right are the same for a given frequency and reflect the time and spatial resolutions. The black bar represents the smallest synthesized beam size at $240 \mathrm{MHz}$ (corresponding to the lower-left points), and the gray bar represents the largest beam size at $80 \mathrm{MHz}$ (corresponding to the upper-right points).

frequency can be straightforwardly converted into a radial height given a density model $n_{e}(r)$, and $d f / d t$ then becomes $d r / d t$. The literature includes a wide range of values using this technique, reflecting the variability among models as well as any intrinsic variability in electron speed. Modest fractions of light speed are typically inferred from drift rates of coronal bursts ( 0.1-0.4 c; Alvarez and Haddock 1973; Aschwanden et al. 1995; Meléndez et al. 1999; Kishore et al. 2017), though speeds larger than 0.5 c have been reported by some studies (Poquerusse, 1994; Klassen, Karlický, and Mann, 2003; Carley, Vilmer, and Gallagher, 2016). Our imaging observations allow us to measure the exciter speed without assuming $n_{e}(r)$ by following the apparent height progression of type III sources at different frequencies.

As in the previous section, we obtain radial heights from centroid positions at the onset of burst emission for each frequency. These data are plotted in Figure 8 along with linear least-squares fits to the speed using the time and spatial resolutions as uncertainties. The 2014-10-14 event exhibits an anomalously late onset time at $80 \mathrm{MHz}$ (see the circles in Figure $3 \mathrm{a}$ and the orange asterisk in Figure 8). This is likely due to the diminished intensity at that frequency, which precludes an appropriate comparison to the onset times at higher frequencies where the burst is much more intense. Figure 8 shows fits both including $(0.29$ c) and excluding $(0.60 \mathrm{c})$ the $80 \mathrm{MHz}$ point for the 2014-10-14 event, and the latter value is used in the discussion to follow because of the better overall fit. Note that while the onset of $80 \mathrm{MHz}$ emission is at a later time than expected given the prior frequency progression, the source location is consistent with the other channels and thus its inclusion not does not impact the inferred density profile from Figures 6 and 7 .

We find an average speed across events of $0.39 \mathrm{c}$, which is consistent with results from other imaging observations. The same strategy was recently employed at lower frequencies by Morosan et al. (2014), who found an average of 0.45 c. McCauley et al. (2017) indirectly inferred a beam speed of 0.2 c from MWA imaging. Chen et al. (2013) also tracked centroid 
Table 1. Imaging Beam Speeds vs $d f / d t$ Model Predictions

\begin{tabular}{l|c|ccc|c}
\hline \multirow{2}{*}{ Event } & \multicolumn{5}{|c}{ Beam Speed (c) } \\
& Imaging & Goryaev et al. & Newkirk & Saito et al. & Cairns et al. $^{b}$ \\
\hline $2014-10-14^{c}$ & $0.60 \pm 0.13$ & $0.38-0.45$ & $0.22-0.31$ & $* * *-0.30$ & 0.58 \\
$2015-09-23$ & $0.24 \pm 0.10$ & $0.34-0.40$ & $0.20-0.28$ & $* * *-0.27$ & 0.50 \\
$2015-10-27$ & $0.32 \pm 0.12$ & $0.44-0.55$ & $0.26-0.36$ & $* * *-0.48$ & 0.40 \\
\hline
\end{tabular}

${ }^{a} f_{p}$ case not viable because model does not include densities above $f_{p} \approx 116 \mathrm{MHz}$.

${ }^{b}$ Model normalized to match the densities implied by our $240 \mathrm{MHz}$ heights.

${ }^{c}$ Excludes the $80 \mathrm{MHz}$ outlier (orange asterisk in Fig. 10).

positions at higher frequencies, though in projection across the disk, finding $0.3 \mathrm{c}$. Mann et al. (2018) recently examined the apparent speeds of three temporally adjacent type III bursts imaged by LOFAR. They find that the sources do not propagate with uniform speed, with each burst exhibiting an acceleration in apparent height, and they conclude that the exciting electron beams must have broad velocity distributions. From Figure 8, we observe an apparent acceleration only for one event (2015-10-27), with the other two events exhibiting the opposite trend to some extent. However, our data are consistent with Mann et al. (2018) in that a uniform speed is not a particularly good fit for any of our events, but the MWA's 0.5 s temporal resolution limits our ability to characterize the source speeds in great detail.

Taken together, we see that speeds measured from imaging observations tend to produce values at the higher end of what is typical for $d f / d t$ inferences. We compare the two approaches for the same events in Table 1 using the same models shown in Figure 6 . We also include speeds derived using the Cairns et al. (2009) model, normalized to the densities implied by our $240 \mathrm{MHz}$ source heights. These values are separated from the others in Table 1 because the normalization precludes direct comparisons to the other models. The $d f / d t$-inferred speeds are consistently smaller than the imaging results for the 2014-10-14 event, which was also true for the bursts studied by Morosan et al. (2014), but there is no major difference between the two approaches for our other events given the range of values. Note that this comparison is arguably a less direct version of the height versus density comparison from the previous section in that the extent to which the imaging and model-dependent $d f / d t$ speeds agree unsurprisingly mirrors the extent to which the density profiles themselves agree. The 2014-10-14 speeds are closest to those derived using $n_{e}(r)$ from Goryaev et al., and the 2015-10-27 result is closest to the Newkirk-derived speed, both assuming harmonic emission, because those density profiles are most closely matched in Figure 6. Likewise, the speeds from those events agree well with $d f / d t$ speeds obtained using the normalized Cairns et al. curves because a $C(r-1)^{-2}$ gradient fits those data nicely. The 2015-09-23 speed is between the two values derived using the Newkirk model assuming either fundamental and harmonic emission, but this may be coincidence given that the modeled and observed density profiles are widely discrepant. That event's non-radial profile may also prevent meaningful agreement with any simple $n_{e}(r)$ model (see Figure 4).

\subsection{Propagation Effects}

As described in Section 1, a number of authors have argued that radio propagation effects, namely refraction and scattering, can explain the large source heights frequently exhibited 
by type III bursts. Bougeret and Steinberg (1977) introduced the idea of scattering by overdense fibers in the context of radio burst morphologies, and Stewart (1974) suggested that type III emission may be produced in underdense flux tubes as a way of explaining observed harmonic-fundamental ratios. These two concepts were combined by Duncan (1979), who introduced the term ducting to refer to radiation that is produced in an underdense environment and subsequently guided to a larger height by reflections against a surrounding "wall" of much higher-density material, which eventually becomes transparent with sufficient altitude. While plausible, this concept generalizes poorly in that electron beams are not expected to be found preferentially within coherent sets of low-density structures that would be conducive to ducting.

Robinson (1983) addressed this by showing that random reflections against overdense fibers can have the same effect of elevating an observed burst site above its true origin, but without requiring any peculiarities of the emission site (i.e. low-density). Because the high density fibers known to permeate the corona are not randomly arranged and are generally radial, random scattering against them does not randomly modulate the aggregate ray path-scattering instead tends to guide the emission outward to larger heights in a manner that is analogous to the classic ducting scenario. For this reason, other authors (e.g. Poquerusse et al. 1988) have chosen to retain ducting to refer to the similar but more general impact of scattering, without implying that the emission is guided within a particular density structure as originally proposed by Duncan (1979). Here, we will simply refer to scattering to avoid potential confusion between the two concepts.

After being scattered for the last time upon reaching a height with sufficiently-low densities, a radio wave will then be refracted through the corona before reaching an observer, further shifting the source location. As the coronal density gradient generally decreases radially, radio waves will tend to refract toward to the radial direction such that a source originating at the limb will appear at a somewhat lower height than its origin, which could be either the actual emission site (e.g. Stewart 1976) or, more likely, the point of last scatter (e.g. Mann et al. 2018). Accounting for the refractive shift, which becomes larger with decreasing frequency, therefore requires that the emission be generated at or scattered to an even larger height than is implied by the observed source location. Recent results on this topic from Mann et al. (2018) will be discussed in the next section.

Propagation effects are also thought to be important to the observed structure of the quiescent corona, where the dominant emission mechanism is thermal bremsstrahlung (freefree) radiation at MWA frequencies. Outside of coronal holes, this emission is expected to be in or close to the optically thick regime (e.g. Kundu 1982; Gibson et al. 2016), which means that the observed brightness temperature should be the same as the coronal temperature. However, well-calibrated 2D measurements have generally found lower brightness temperatures than expected from temperatures derived at other wavelengths (see review by Lantos 1999). Additionally, the size of the corona appears to be larger than expected at low frequencies (e.g. Aubier, Leblanc, and Boischot 1971; Thejappa and Kundu 1992; Sastry 1994; Subramanian 2004; Ramesh et al. 2006). The prevailing explanation for these effects is also scattering by density inhomogeneities (e.g. Melrose and Dulk 1988; Alissandrakis 1994; Thejappa and MacDowall 2008), though the refractive effect described in the previous paragraph is also important (Thejappa and MacDowall, 2008).

Thus, the scattering process that may act to elevate type III sources also affects quiescent emission, increasing the apparent size of the corona. We will take advantage of this by using the difference in extent between observed and modeled quiescent emission as a proxy for the net effect of propagation effects on our type III source heights. This approach is limited in 


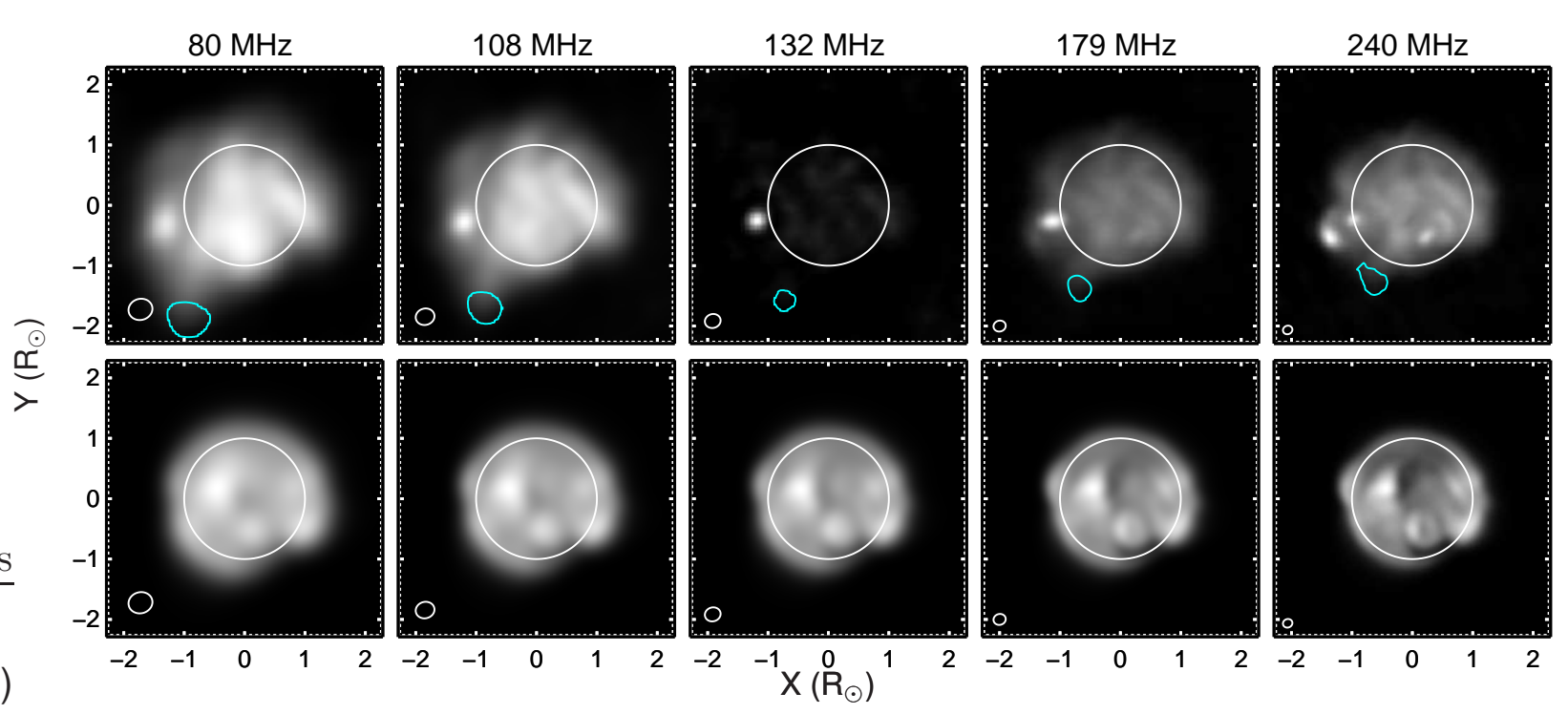

Figure 9. MWA background images for the 2014-10-14 event (top) and corresponding MAS-FORWARD synthetic images convolved with the MWA beam (bottom). Beam ellipses are shown in the lower-left corners, and the cyan curves are the $50 \%$ burst contours from Figs. $1 \& 4$. This day is shown because thermal emission is only barely distinguishable at $132 \mathrm{MHz}$, precluding the Fig. 10 analysis at that frequency, which was not the case for any other event-channel combination.
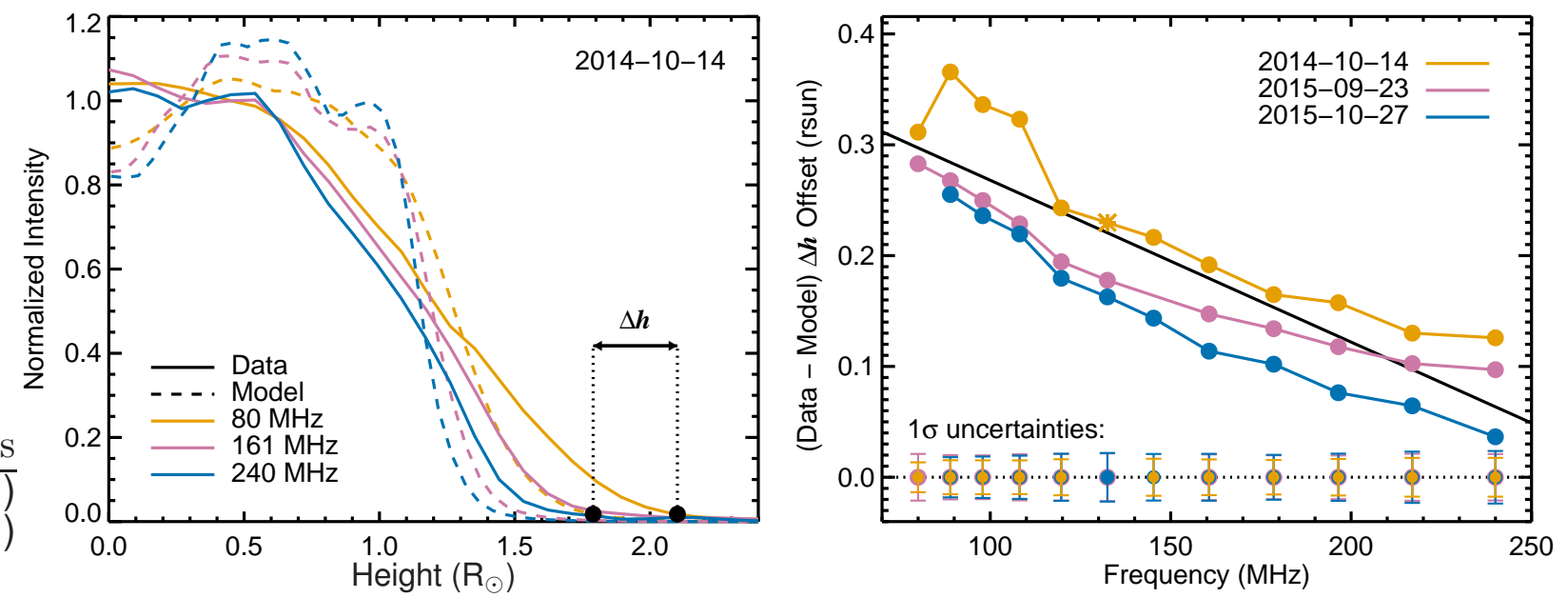

Figure 10. Left: Average intensity versus radial distance obtained from the Fig. 9 images and normalized by the median value below $1 \mathrm{R} \odot . \Delta h$ refers to the height offset between the observed and modeled intensity profiles at the apparent type III burst height at $80 \mathrm{MHz}$. Right: $\Delta h$ for each frequency and event. An orange asterisk marks the one instance where data was available but a measurement could not be made because the thermal background emission was not well-detected (see Fig. 9), so an average of the adjacent points is used. The uncertainties reflect the sensitivity of $\Delta h$ to the normalization choice in the left panel (see Sec. 3.3).

that, although both are related to scattering, the extent to which the magnitudes of these two phenomena are related is unclear. In particular, previous studies on the broadening of the radio Sun by scattering have considered random density inhomogeneities as opposed to the more realistic case of high density fibers capable of producing the ducting-like effect for type III sources.

Figure 9 shows the observed background emission versus synthetic images based on a global MHD model. The MWA images are obtained by averaging every frame with a total intensity less than $2 \sigma$ above the background level, which is determined via the procedure shown in Figure 5. Synthetic images are obtained using FORWARD (Gibson et al., 2016), 
a software suite that calculates the expected bremsstrahlung and gyroresonance emission given a model atmosphere. We use the Magnetohydrodynamic Algorithm outside a Sphere (MAS; Lionello, Linker, and Mikić 2009) medium resolution (hmi_mast_mas_std_0201) model, which extrapolates the coronal magnetic field from photospheric magnetograms (e.g. Mikić et al. 1999) and applies a heating model adapted from Schrijver et al. (2004) to compute density and temperature.

McCauley et al. (2017) established the use of these model images for flux calibration and included a qualitative comparison to MWA observations. As in the aforementioned literature, the radial extent of the corona is somewhat larger in the observations than in the beam-convolved model images. To quantify this difference, we divide both image sets into concentric rings about Sun-center. The average intensity within each ring is plotted against its radial distance in the left panel of Figure 10, where the intensities have been normalized by the median value below $1 \mathrm{R}_{\odot}$. We then measure the offset $\Delta h$ between the observed and modeled profiles at the heights obtained from the type III positions. $\Delta h$ is sensitive to how the intensity curves are normalized, and we quantify this uncertainty by repeating the procedure for 10 different normalization factors that reflect the median intensities within radial bins of width $0.1 \mathrm{R}_{\odot}$ from 0 to $1 \mathrm{R}_{\odot}$. The right panel of Figure 10 plots the $\Delta h$ results for each event, which have $1 \sigma$ uncertainties of less than $\pm 0.025 \mathrm{R}_{\odot}$. The offset appears to depend roughly linearly on frequency, with larger offsets at lower frequencies. Fitting a line through all of the points, we find that:

$$
\Delta h \approx-1.5 \times 10^{-3} f+0.41 ; 80 \leq f \leq 240 \mathrm{MHz}
$$

where $\Delta h$ is in solar radii and $f$ is in MHz. This yields $0.30 \mathrm{R}_{\odot}$ at $80 \mathrm{MHz}$ and $0.06 \mathrm{R}_{\odot}$ at $240 \mathrm{MHz}$. We do not expect this expression to be relevant much outside of the prescribed frequency range, but extrapolating slightly, we obtain $0.32 \mathrm{R}_{\odot}$ at $60 \mathrm{MHz}$. This value is a bit more than half of the $<0.56 \mathrm{R}_{\odot}$ limit found by Poquerusse et al. (1988).

Poquerusse et al., and others who have quantified the scattering effect (e.g. Robinson 1983), obtained their results by computing ray trajectories through a model corona. That approach allows a fuller understanding of the propagation physics, but the result is dependent on the assumed concentration and distribution of high density fibers, which are not well constrained. Our critical assumption is that emission produced at significantly lower heights would be absorbed, as would be the case in our optically-thick model corona. However, low coronal brightness temperatures could also be explained by lower opacities (e.g. Mercier and Chambe 2009) or a low filling factor, which would allow burst emission to escape from lower heights and lead us to underestimate the potential impact of propagation effects.

Figure 11 shows how the Figure 6 density results change after subtracting the height offsets from Figure 10. The 2014-10-14 harmonic $\left(2 f_{p}\right)$ profile remains reasonable with the offsets, lying just below the Goryaev et al. (2014) model instead of just above it, while the fundamental emission densities for that event would still be quite large. Given that the Goryaev et al. model is among the highest-density streamer models in the literature, we conclude that harmonic emission from a beam traveling along an overdense structure is consistent with 2014-10-14 data. Our assessment for this event is that propagation effects may contribute to some but not all the apparent density enhancement.

The other two events exhibit unusually steep density profiles once the offsets are subtracted. That was true also for the original 2015-09-23 results, which we attributed to its non-radial trajectory in Section 3.1. However, the original 2015-10-27 densities were 


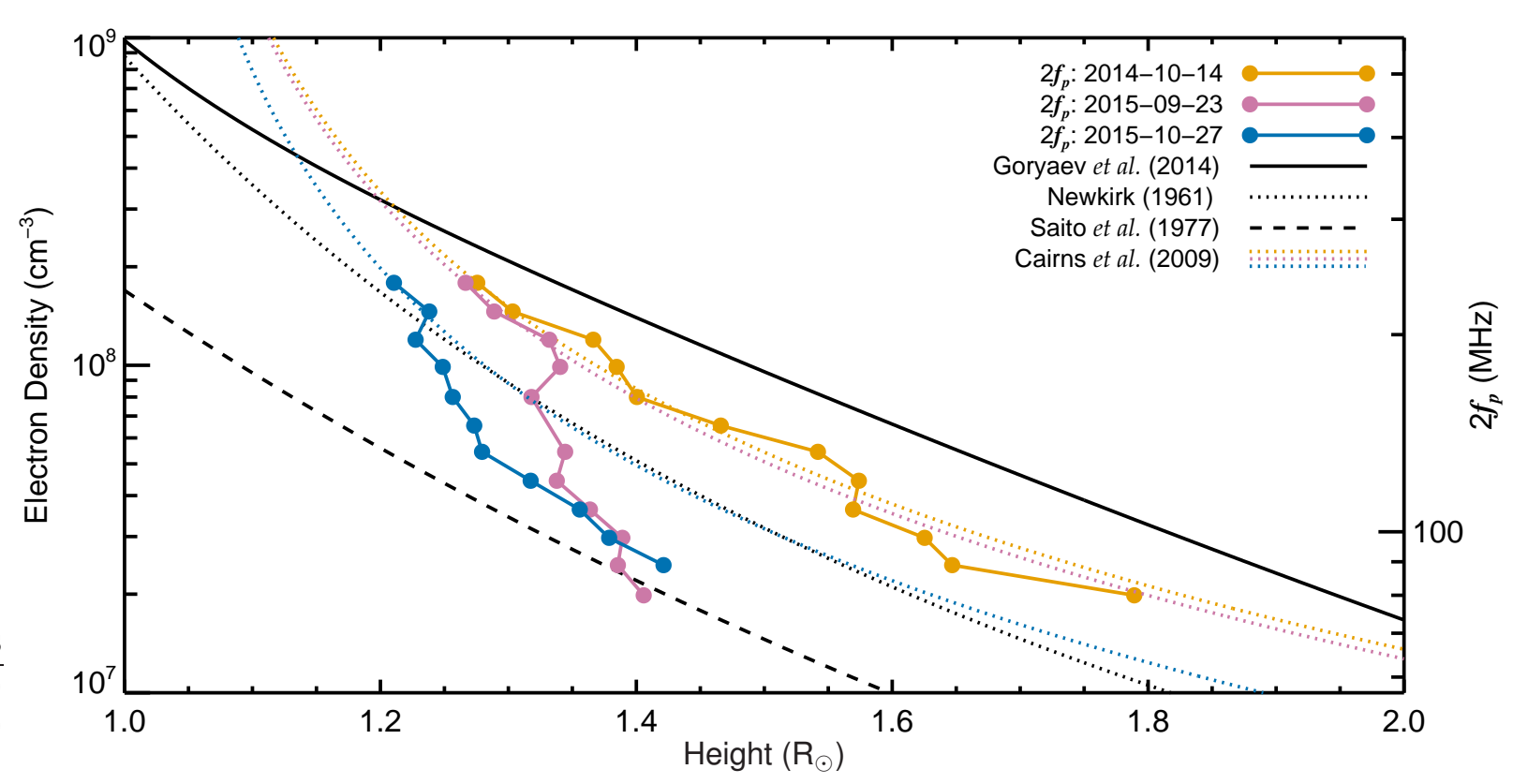

Figure 11. Imaging density profiles after applying the $\Delta h$ offsets from Fig. 10 and assuming harmonic emission. Model curves are as in Figs. $6 \& 7$.

well-matched to the Cairns et al. (2009) $C(r-1)^{-2}$ gradient but become too steep to match any standard density gradient with the inferred offsets. This may simply reflect the intrinsic density gradient of the particular structure. Alternatively, it is possible that we have over- or underestimated the impact of propagation effects at the low or high end of our frequency range, respectively. However, the frequency dependence of scattering and refraction means that any treatment will steepen the density gradient. Aside from their slopes, the offsets bring the densities implied by both bursts to generally within the normal background range assuming harmonic emission or to a moderately enhanced level assuming fundamental emission.

\section{Discussion}

The previous section suggests that propagation effects can partially explain the apparent density enhancements implied by our type III source heights. Assuming harmonic emission, our estimates for the potential magnitude of propagation effects bring the densities to within normal background levels for two events, while one event remains enhanced at a level consistent with a dense streamer. In Section 3.1, we showed that our original density profiles are consistent with those found from recent type III burst studies at lower and higher frequencies, which together are well-fit by the Cairns et al. (2009) $C(r-1)^{-2}$ gradient. Both the low- and high-frequency studies conclude that their large densities imply electron beams traveling along overdense structures, but neither consider the impact of propagation effects.

Morosan et al. (2014) propose a variation of the overdense hypothesis based on their 30-60 MHz LOFAR observations. They suggest that the passage of a CME just prior to an electron beam's arrival may compress streamer plasma enough to facilitate type III emission at unusually large heights. This was consistent with their events being associated with a CME and could be relevant to our 2015-09-23 event, which was also preceded by a CME (see Section 2.3). While this interpretation is plausible, we do not think such special conditions need to be invoked given that the densities inferred from the Morosan et al. 
results are consistent with ours (Figure 7) and are broadly consistent with the large type III source heights found using the previous generations of low frequency instruments (e.g. Wild, Sheridan, and Neylan 1959; Morimoto 1964; Malitson and Erickson 1966; Stewart 1976; Kundu and Stone 1984). Propagation effects seem particularly likely to have contributed (at least partially) to their inferred density enhancement, as the effects become stronger with decreasing frequency.

Recently, Mann et al. (2018) also examined the heights of type III sources observed at the limb by LOFAR. After accounting for the refractive effect described in Section 3.3, and relying on scattering to direct emission toward the observer at large heights prior to being refracted, their results imply a density enhancement of around $3.3 \times$ over the Newkirk (1961) density model, assuming fundamental emission. Incorporating our offsets from Section 3.3 gives us an average enhancement of $4.6 \times$ over the same model across our three events, also assuming fundamental emission. Our results are therefore consistent with those of Mann et al. (2018), though our attempts quantify the impact of propagation effects are quite different.

Chen et al. (2013) also found large densities using VLA data at higher frequencies $(1.0-1.5 \mathrm{GHz})$. Scattering is also thought to be important at high frequencies given the apparent lack of small-scale structure (Bastian, 1994), but the extent to which scattering may also elevate radio sources in that regime has not been addressed to our knowledge. Chen et al. observed an on-disk event, from which they obtain source heights by comparing their projected positions to stereoscopic observations of an associated EUV jet, which is assumed to have the same inclination as the type III electron beam. This method would also be impacted by any source shifting caused by scattering. Although these shifts would be much smaller than at low frequencies, the background gradient is much steeper, so a reasonably small shift may still strongly influence the inferred density relative to the background.

If we accept the densities obtained at higher frequencies, albeit from just one example, then their consistency with low frequency observations in general is striking. As we describe in Section 1, the community largely came to favor propagation effects over the overdense hypothesis in the 1980s, and the topic has not had much consideration since. If new observations at low heights (high frequencies) also suggest beams moving preferentially along dense structures, then it begs the question of whether or not that interpretation is again viable at larger heights (lower frequencies). In that case, this would need to be reconciled with the fact that electron beams have not been found to be preferentially associated with particularly high density regions in in situ solar wind measurements (Steinberg et al., 1984), along with the evidence for other impacts of scattering such as angular broadening (e.g. Steinberg, Hoang, and Dulk 1985; Bastian 1994; Ingale, Subramanian, and Cairns 2015).

Selection effects may be relevant, as radiation produced well above the ambient plasma frequency is less likely to be absorbed before reaching the observer. Thus, coronal type III bursts may imply high densities because beams traveling along dense structures are likelier to be observed. Type III bursts also have a range of starting frequencies, which has been interpreted in terms of a range in acceleration (i.e. reconnection) heights that are often larger than those inferred from X-ray observations (Reid, Vilmer, and Kontar, 2014). Alternatively, a beam may be accelerated at a smaller height than is implied by the resultant type III starting frequency due to unfavorable radiation escape conditions (absorption) below the apparent starting height. Simulations also suggest that electron beams may travel a significant distance before producing observable emission ( $\mathrm{Li}$ and 
Cairns, 2013, 2014) and that they may be radio loud at some frequencies but not at others due to variations in the ambient density (Li, Cairns, and Robinson, 2012; Loi, Cairns, and $\mathrm{Li}, 2014$ ) and/or temperature (Li, Cairns, and Robinson, 2011a,b).

The magnetic structures along which electron beams travel also evolve with distance from the Sun. A popular open flux tube model is an expanding funnel that is thin at the base of the corona and increasingly less so into the solar wind (e.g. Byhring, Esser, and Lie-Svendsen 2008; He, Tu, and Marsch 2008; Pucci, Lie-Svendsen, and Esser 2010). Such structures may allow a dense flux tube to become less dense relative to the background as it expands with height. Moreover, a beam following a particular magnetic field line from the corona into the high- $\beta$ solar wind may not necessarily encounter a coherent density structure throughout the heliosphere. Turbulent mixing, corotation interaction regions, CMEs, and other effects influence solar wind density such that it is not obvious that an electron beam traversing an overdense structure near the Sun should also be moving in an overdense structure at large heliocentric distances.

We also note that one of the main conclusions from many of the type III studies referenced here is unchanged in either the overdense or propagation effects scenarios. Both cases require a very fibrous corona that can supply dense structures along which beams may travel and/or dense structures capable of scattering radio emission to larger heights. This consistent with the fine structure known from eclipse observations (e.g. Woo 2007) that has more recently been evidenced by EUV observations. For instance, analyses of a sungrazing comet (Raymond et al., 2014) and of EUV spectra (Hahn and Savin, 2016) independently suggest large density contrasts $(\gtrsim 3-10)$ between neighboring flux tubes in regions where the structures themselves are undetected. As our understanding of such fine structure improves, better constraints can be placed on them for the purpose of modeling the impact of propagation effects on radio sources.

\section{Conclusion}

We presented imaging of three isolated type III bursts observed at the limb on different days using the MWA. Each event is associated with a white light streamer and plausibly associated with EUV rays that exhibit activity around the time of the radio bursts. Assuming harmonic plasma emission, density profiles derived from the source heights imply enhancements of $\sim 2.4-5.4 \times$ over background levels. This corresponds to electron densities of $1.8 \times 10^{8} \mathrm{~cm}^{-3}(240 \mathrm{MHz})$ down to $0.20 \times 10^{8} \mathrm{~cm}^{-3}(80 \mathrm{MHz})$ at average heights of 1.3 to $1.9 \mathrm{R}_{\odot}$. These values are consistent with the highest streamer densities inferred from other wavelengths and with the large radio source heights found using older instruments. The densities are also consistent with recent type III results at higher and lower frequencies, which combined are well-fit by a $C(r-1)^{-2}$ gradient. By comparing the extent of the radio limb to model predictions, we estimated that radio propagation effects, principally the ducting-like effect of random scattering by high density fibers, may be responsible for $0.06-0.30 \mathrm{R}_{\odot}$ of our apparent source heights. This shift brings the results from 2 of our 3 events to within a standard range of background densities. We therefore conclude that propagation effects can partially explain the apparent density enhancements but that beams moving along overdense structures cannot be ruled out. We also used the imaging data to estimate electron beam speeds of $0.24-0.60 \mathrm{c}$.

Acknowledgments Support for this work was provided by the Australian Government through an Endeavour Postgraduate Scholarship. We thank Stephen White and Don Melrose for helpful discussions 
and the anonymous referee for their constructive comments. This scientific work makes use of the Murchison Radio-astronomy Observatory (MRO), operated by the Commonwealth Scientific and Industrial Research Organisation (CSIRO). We acknowledge the Wajarri Yamatji people as the traditional owners of the Observatory site. Support for the operation of the MWA is provided by the Australian Government's National Collaborative Research Infrastructure Strategy (NCRIS), under a contract to Curtin University administered by Astronomy Australia Limited. We acknowledge the Pawsey Supercomputing Centre, which is supported by the Western Australian and Australian Governments. The SDO is a National Aeronautics and Space Administration (NASA) satellite, and we acknowledge the AIA science team for providing open access to data and software. The SOHO/LASCO data used here are produced by a consortium of the Naval Research Laboratory (USA), Max-Planck-Institut fuer Aeronomie (Germany), Laboratoire d'Astronomie (France), and the University of Birmingham (UK). SOHO is a project of international cooperation between ESA and NASA. This research has also made use of NASA's Astrophysics Data System (ADS), along with JHelioviewer (Müller et al., 2017) and the Virtual Solar Observatory (VSO, Hill et al. 2009).

\section{References}

Alissandrakis, C.E.: 1994, Radio observations of the quiet solar corona. Advances in Space Research 14. DOI. ADS.

Allen, C.W.: 1947, Interpretation of Electron Densities from Corona Brightness. Mon. Not. Roy. Astron. Soc. 107, 426. DOI. ADS.

Alvarez, H., Haddock, F.T.: 1973, Solar Wind Density Model from km-Wave Type III Bursts. Solar Phys. 29, 197. DOI. ADS.

Aschwanden, M.J., Benz, A.O., Dennis, B.R., Schwartz, R.A.: 1995, Solar Electron Beams Detected in Hard X-Rays and Radio Waves. Astrophys. J. 455, 347. DOI. ADS.

Aubier, M., Leblanc, Y., Boischot, A.: 1971, Observations of the Quiet Sun at Decameter Wavelengths Effects of Scattering on the Brightness Distribution. Astron. Astrophys. 12, 435. ADS.

Bastian, T.S.: 1994, Angular scattering of solar radio emission by coronal turbulence. Astrophys. J. 426, 774. DOI. ADS.

Bastian, T.S., Benz, A.O., Gary, D.E.: 1998, Radio Emission from Solar Flares. Ann. Rev. Astron. Astrophys. 36, 131. DOI. ADS.

Benz, A.O., Brajša, R., Magdalenić, J.: 2007, Are There Radio-quiet Solar Flares? Solar Phys. $240,263$. DOI. ADS.

Benz, A.O., Grigis, P.C., Csillaghy, A., Saint-Hilaire, P.: 2005, Survey on Solar X-ray Flares and Associated Coherent Radio Emissions. Solar Phys. 226, 121. DOI. ADS.

Bisoi, S.K., Sawant, H.S., Janardhan, P., Yan, Y., Chen, L., Awasthi, A.K., Srivastava, S., Gao, G.: 2018, Decimetric emission 500" away from a flaring Site: Possible scenarios from GMRT solar radio observations. ArXiv e-prints. ADS.

Bougeret, J.L., Steinberg, J.L.: 1977, A new scattering process above solar active regions - Propagation in a fibrous medium. Astron. Astrophys. 61, 777. ADS.

Bougeret, J.-L., King, J.H., Schwenn, R.: 1984, Solar radio burst and in situ determination of interplanetary electron density. Solar Phys. 90, 401. DOI. ADS.

Briggs, D.S.: 1995, High Fidelity Interferometric Imaging: Robust Weighting and NNLS Deconvolution. In: American Astronomical Society Meeting Abstracts, Bulletin of the American Astronomical Society 27, 1444. ADS.

Brueckner, G.E., Howard, R.A., Koomen, M.J., Korendyke, C.M., Michels, D.J., Moses, J.D., Socker, D.G., Dere, K.P., Lamy, P.L., Llebaria, A., Bout, M.V., Schwenn, R., Simnett, G.M., Bedford, D.K., Eyles, C.J.: 1995, The Large Angle Spectroscopic Coronagraph (LASCO). Solar Phys. 162, 357. DOI. ADS.

Byhring, H.S., Esser, R., Lie-Svendsen, Ø.: 2008, The Funnel Geometry of Open Flux Tubes in the Low Solar Corona Constrained by O VI and Ne VIII Outflow. Astrophys. J. Lett. 673, L91. DOI. ADS.

Cairns, I.H., Lobzin, V.V., Warmuth, A., Li, B., Robinson, P.A., Mann, G.: 2009, Direct Radio Probing and Interpretation of the Sun's Plasma Density Profile. Astrophys. J. Lett. 706, L265. DOI. ADS. 
Cairns, I.H., Lobzin, V.V., Donea, A., Tingay, S.J., McCauley, P.I., Oberoi, D., Duffin, R.T., Reiner, M.J., Hurley-Walker, N., Kudryavtseva, N.A., Melrose, D.B., Harding, J.C., Bernardi, G., Bowman, J.D., Cappallo, R.J., Corey, B.E., Deshpande, A., Emrich, D., Goeke, R., Hazelton, B.J., JohnstonHollitt, M., Kaplan, D.L., Kasper, J.C., Kratzenberg, E., Lonsdale, C.J., Lynch, M.J., McWhirter, S.R., Mitchell, D.A., Morales, M.F., Morgan, E., Ord, S.M., Prabu, T., Roshi, A., Shankar, N.U., Srivani, K.S., Subrahmanyan, R., Wayth, R.B., Waterson, M., Webster, R.L., Whitney, A.R., Williams, A., Williams, C.L.: 2018, Low Altitude Solar Magnetic Reconnection, Type III Solar Radio Bursts, and X-ray Emissions. Scientific Reports 8, 1676. DOI. ADS.

Cane, H.V., Erickson, W.C., Prestage, N.P.: 2002, Solar flares, type III radio bursts, coronal mass ejections, and energetic particles. Journal of Geophysical Research (Space Physics) 107, 1315. DOI. ADS.

Carley, E.P., Vilmer, N., Gallagher, P.T.: 2016, Radio Diagnostics of Electron Acceleration Sites During the Eruption of a Flux Rope in the Solar Corona. Astrophys. J. 833, 87. DOI. ADS.

Chen, B., Bastian, T.S., White, S.M., Gary, D.E., Perley, R., Rupen, M., Carlson, B.: 2013, Tracing Electron Beams in the Sun's Corona with Radio Dynamic Imaging Spectroscopy. Astrophys. J. Lett. 763, L21. DOI. ADS.

Cliver, E.W., Ling, A.G.: 2009, Low-Frequency Type III Bursts and Solar Energetic Particle Events. Astrophys. J. 690, 598. DOI. ADS.

Domingo, V., Fleck, B., Poland, A.I.: 1995, SOHO: The Solar and Heliospheric Observatory. Space Sci. Rev. 72, 81. DOI. ADS.

Dulk, G.A.: 2000, Type III Solar Radio Bursts at Long Wavelengths. Washington DC American Geophysical Union Geophysical Monograph Series 119, 115. DOI. ADS.

Dulk, G.A., Suzuki, S.: 1980, The position and polarization of Type III solar bursts. Astron. Astrophys. 88, 203. ADS.

Duncan, R.A.: 1979, Wave ducting of solar metre-wave radio emission as an explanation of fundamental/harmonic source coincidence and other anomalies. Solar Phys. 63, 389. DOI. ADS.

Fainberg, J., Stone, R.G.: 1971, Type III Solar Radio Burst Storms Observed at Low Frequencies. III: Streamer Density, Inhomogeneities, and Solar Wind Speed. Solar Phys. 17, 392. DOI. ADS.

Freeland, S.L., Handy, B.N.: 1998, Data Analysis with the SolarSoft System. Solar Phys. 182, 497. DOI. ADS.

Gibson, S.E., Fludra, A., Bagenal, F., Biesecker, D., del Zanna, G., Bromage, B.: 1999, Solar minimum streamer densities and temperatures using Whole Sun Month coordinated data sets. J. Geophys. Res. 104, 9691. DOI. ADS.

Gibson, S., Kucera, T., White, S., Dove, J., Fan, Y., Forland, B., Rachmeler, L., Downs, C., Reeves, K.: 2016, FORWARD: A toolset for multiwavelength coronal magnetometry. Frontiers in Astronomy and Space Sciences 3, 8. DOI. ADS.

Ginzburg, V.L., Zhelezniakov, V.V.: 1958, On the Possible Mechanisms of Sporadic Solar Radio Emission (Radiation in an Isotropic Plasma). Soviet Astron. 2, 653. ADS.

Gopalswamy, N., Kundu, M.R., Szabo, A.: 1987, Propagation of electrons emitting weak type III bursts in coronal streamers. Solar Phys. 108, 333. DOI. ADS.

Goryaev, F., Slemzin, V., Vainshtein, L., Williams, D.R.: 2014, Study of Extreme-ultraviolet Emission and Properties of a Coronal Streamer from PROBA2/SWAP, Hinode/EIS and Mauna Loa Mk4 Observations. Astrophys. J. 781, 100. DOI. ADS.

Guhathakurta, M., Holzer, T.E., MacQueen, R.M.: 1996, The Large-Scale Density Structure of the Solar Corona and the Heliospheric Current Sheet. Astrophys. J. 458, 817. DOI. ADS.

Guidice, D.A., Cliver, E.W., Barron, W.R., Kahler, S.: 1981, The Air Force RSTN System. In: Bulletin of the American Astronomical Society, Bull. Am. Astron. Soc. 13, 553. ADS.

Hahn, M., Savin, D.W.: 2016, Inferring the Coronal Density Irregularity from EUV Spectra. Astrophys. J. 829, 42. DOI. ADS.

He, J.-S., Tu, C.-Y., Marsch, E.: 2008, Modeling of Solar Wind in the Coronal Funnel with Mass and Energy Supplied at $5 \mathrm{Mm}$. Solar Phys. 250, 147. DOI. ADS.

Hill, F., Martens, P., Yoshimura, K., Gurman, J., Hourclé, J., Dimitoglou, G., Suárez-Solá, I., Wampler, S., Reardon, K., Davey, A., Bogart, R.S., Tian, K.Q.: 2009, The Virtual Solar Observatory-A Resource 
for International Heliophysics Research. Earth Moon and Planets 104, 315. DOI. ADS.

Howard, R.A., Moses, J.D., Vourlidas, A., Newmark, J.S., Socker, D.G., Plunkett, S.P., Korendyke, C.M., Cook, J.W., Hurley, A., Davila, J.M., Thompson, W.T., St Cyr, O.C., Mentzell, E., Mehalick, K., Lemen, J.R., Wuelser, J.P., Duncan, D.W., Tarbell, T.D., Wolfson, C.J., Moore, A., Harrison, R.A., Waltham, N.R., Lang, J., Davis, C.J., Eyles, C.J., Mapson-Menard, H., Simnett, G.M., Halain, J.P., Defise, J.M., Mazy, E., Rochus, P., Mercier, R., Ravet, M.F., Delmotte, F., Auchere, F., Delaboudiniere, J.P., Bothmer, V., Deutsch, W., Wang, D., Rich, N., Cooper, S., Stephens, V., Maahs, G., Baugh, R., McMullin, D., Carter, T.: 2008, Sun Earth Connection Coronal and Heliospheric Investigation (SECCHI). Space Sci. Rev. 136, 67. DOI. ADS.

Hurley-Walker, N., Morgan, J., Wayth, R.B., Hancock, P.J., Bell, M.E., Bernardi, G., Bhat, R., Briggs, F., Deshpande, A.A., Ewall-Wice, A., Feng, L., Hazelton, B.J., Hindson, L., Jacobs, D.C., Kaplan, D.L., Kudryavtseva, N., Lenc, E., McKinley, B., Mitchell, D., Pindor, B., Procopio, P., Oberoi, D., Offringa, A., Ord, S., Riding, J., Bowman, J.D., Cappallo, R., Corey, B., Emrich, D., Gaensler, B.M., Goeke, R., Greenhill, L., Hewitt, J., Johnston-Hollitt, M., Kasper, J., Kratzenberg, E., Lonsdale, C., Lynch, M., McWhirter, R., Morales, M.F., Morgan, E., Prabu, T., Rogers, A., Roshi, A., Shankar, U., Srivani, K., Subrahmanyan, R., Tingay, S., Waterson, M., Webster, R., Whitney, A., Williams, A., Williams, C.: 2014, The Murchison Widefield Array Commissioning Survey: A Low-Frequency Catalogue of 14110 Compact Radio Sources over 6100 Square Degrees. PASA 31, e045. DOI. ADS.

Hurley-Walker, N., Callingham, J.R., Hancock, P.J., Franzen, T.M.O., Hindson, L., Kapińska, A.D., Morgan, J., Offringa, A.R., Wayth, R.B., Wu, C., Zheng, Q., Murphy, T., Bell, M.E., Dwarakanath, K.S., For, B., Gaensler, B.M., Johnston-Hollitt, M., Lenc, E., Procopio, P., Staveley-Smith, L., Ekers, R., Bowman, J.D., Briggs, F., Cappallo, R.J., Deshpande, A.A., Greenhill, L., Hazelton, B.J., Kaplan, D.L., Lonsdale, C.J., McWhirter, S.R., Mitchell, D.A., Morales, M.F., Morgan, E., Oberoi, D., Ord, S.M., Prabu, T., Shankar, N.U., Srivani, K.S., Subrahmanyan, R., Tingay, S.J., Webster, R.L., Williams, A., Williams, C.L.: 2017, GaLactic and Extragalactic All-sky Murchison Widefield Array (GLEAM) survey - I. A low-frequency extragalactic catalogue. Mon. Not. Roy. Astron. Soc. 464, 1146. DOI. ADS.

Ingale, M., Subramanian, P., Cairns, I.: 2015, Coronal turbulence and the angular broadening of radio sources - the role of the structure function. Mon. Not. Roy. Astron. Soc. 447, 3486. DOI. ADS.

Innes, D.E., Bučík, R., Guo, L.-J., Nitta, N.: 2016, Observations of solar X-ray and EUV jets and their related phenomena. Astronomische Nachrichten 337, 1024. DOI. ADS.

Kennewell, J., Steward, G.: 2003, Solar Radio Spectrograph [SRS] Data Viewer. Technical report, Sydney, IPS Radio and Space Serv..

Kishore, P., Kathiravan, C., Ramesh, R., Ebenezer, E.: 2017, Coronal Magnetic Field Lines and Electrons Associated with Type III-V Radio Bursts in a Solar Flare. Journal of Astrophysics and Astronomy 38, \#24. DOI. ADS.

Klassen, A., Karlický, M., Mann, G.: 2003, Superluminal apparent velocities of relativistic electron beams in the solar corona. Astron. Astrophys. 410, 307. DOI. ADS.

Kontar, E.P., Yu, S., Kuznetsov, A.A., Emslie, A.G., Alcock, B., Jeffrey, N.L.S., Melnik, V.N., Bian, N.H., Subramanian, P.: 2017, Imaging spectroscopy of solar radio burst fine structures. Nature Communications 8, 1515. DOI. ADS.

Koutchmy, S.: 1994, Coronal physics from eclipse observations. Advances in Space Research 14. DOI. ADS.

Krupar, V., Kontar, E.P., Soucek, J., Santolik, O., Maksimovic, M., Kruparova, O.: 2015, On the speed and acceleration of electron beams triggering interplanetary type III radio bursts. Astron. Astrophys. 580, A137. DOI. ADS.

Kundu, M.R.: 1982, Advances in solar radio astronomy. Reports on Progress in Physics 45, 1435. DOI. ADS.

Kundu, M.R., Stone, R.G.: 1984, Observations of solar radio bursts from meter to kilometer wavelengths. Advances in Space Research 4, 261. DOI. ADS.

Lantos, P.: 1999, Low Frequency Observations of the Quiet Sun: a Review. In: Bastian, T.S., Gopalswamy, N., Shibasaki, K. (eds.) Proceedings of the Nobeyama Symposium 479, 11. ADS.

Leblanc, Y.: 1973, Scattering Effects on the Relative Positions and Intensities of Fundamental and Harmonic Emission of Solar Radio Bursts. Astrophys. Lett. 14, 41. ADS. 
Leblanc, Y., de La Noe, J.: 1977, Solar radio type III bursts and coronal density structures. Solar Phys. 52, 133. DOI. ADS.

Leblanc, Y., Dulk, G.A., Bougeret, J.-L.: 1998, Tracing the Electron Density from the Corona to 1 au. Solar Phys. 183, 165. DOI. ADS.

Leblanc, Y., Kuiper, T.B.H., Hansen, S.F.: 1974, Coronal Density Structures in Regions of Type III Activity. Solar Phys. 37, 215. DOI. ADS.

Lecacheux, A., Steinberg, J.-L., Hoang, S., Dulk, G.A.: 1989, Characteristics of type III bursts in the solar wind from simultaneous observations on board ISEE-3 and Voyager. Astron. Astrophys. 217, 237. ADS.

Lemen, J.R., Title, A.M., Akin, D.J., Boerner, P.F., Chou, C., Drake, J.F., Duncan, D.W., Edwards, C.G., Friedlaender, F.M., Heyman, G.F., Hurlburt, N.E., Katz, N.L., Kushner, G.D., Levay, M., Lindgren, R.W., Mathur, D.P., McFeaters, E.L., Mitchell, S., Rehse, R.A., Schrijver, C.J., Springer, L.A., Stern, R.A., Tarbell, T.D., Wuelser, J.-P., Wolfson, C.J., Yanari, C., Bookbinder, J.A., Cheimets, P.N., Caldwell, D., Deluca, E.E., Gates, R., Golub, L., Park, S., Podgorski, W.A., Bush, R.I., Scherrer, P.H., Gummin, M.A., Smith, P., Auker, G., Jerram, P., Pool, P., Soufli, R., Windt, D.L., Beardsley, S., Clapp, M., Lang, J., Waltham, N.: 2012, The Atmospheric Imaging Assembly (AIA) on the Solar Dynamics Observatory (SDO). Solar Phys. 275, 17. DOI. ADS.

Li, B., Cairns, I.H.: 2013, Type III bursts produced by power law injected electrons in Maxwellian background coronal plasmas. Journal of Geophysical Research (Space Physics) 118, 4748. DOI. ADS.

Li, B., Cairns, I.H.: 2014, Fundamental Emission of Type III Bursts Produced in Non-Maxwellian Coronal Plasmas with Kappa-Distributed Background Particles. Solar Phys. 289, 951. DOI. ADS.

Li, B., Cairns, I.H., Robinson, P.A.: 2011a, Effects of Spatial Variations in Coronal Electron and Ion Temperatures on Type III Bursts. II. Variations in Ion Temperature. Astrophys. J. 730, 21. DOI. ADS.

Li, B., Cairns, I.H., Robinson, P.A.: 2011b, Effects of Spatial Variations in Coronal Temperatures on Type III Bursts. I. Variations in Electron Temperature. Astrophys. J. 730, 20. DOI. ADS.

Li, B., Cairns, I.H., Robinson, P.A.: 2012, Frequency Fine Structures of Type III Bursts Due to Localized Medium-Scale Density Structures Along Paths of Type III Beams. Solar Phys. 279, 173. DOI. ADS.

Lionello, R., Linker, J.A., Mikić, Z.: 2009, Multispectral Emission of the Sun During the First Whole Sun Month: Magnetohydrodynamic Simulations. Astrophys. J. 690, 902. DOI. ADS.

Lobzin, V.V., Cairns, I.H., Robinson, P.A., Warmuth, A., Mann, G., Gorgutsa, R.V., Fomichev, V.V.: 2010, Evidence for Gently Sloping Plasma Density Profiles in the Deep Corona: Type III Observations. Astrophys. J. 724, 1099. DOI. ADS.

Loi, S.T., Cairns, I.H., Li, B.: 2014, Production of Fine Structures in Type III Solar Radio Bursts Due to Turbulent Density Profiles. Astrophys. J. 790, 67. DOI. ADS.

Lonsdale, C., Benkevitch, L., Cairns, I., Crowley, M., Erickson, P., Knapp, M., Kozarev, K., Lind, F., McCauley, P., Morgan, J., Oberoi, D.: 2018, Solar Imaging using Low Frequency Arrays. ArXiv e-prints. ADS.

Malitson, H.H., Erickson, W.C.: 1966, Observations of Type III and Type IV Solar Radio Bursts at 26.3 Mc/s. Astrophys. J. 144, 337. DOI. ADS.

Mann, G., Jansen, F., MacDowall, R.J., Kaiser, M.L., Stone, R.G.: 1999, A heliospheric density model and type III radio bursts. Astron. Astrophys. 348, 614. ADS.

Mann, G., Breitling, F., Vocks, C., Aurass, H., Steinmetz, M., Strassmeier, K.G., Bisi, M.M., Fallows, R.A., Gallagher, P., Kerdraon, A., Mackinnon, A., Magdalenic, J., Rucker, H., Anderson, J., Asgekar, A., Avruch, I.M., Bell, M.E., Bentum, M.J., Bernardi, G., Best, P., Bîrzan, L., Bonafede, A., Broderick, J.W., Brüggen, M., Butcher, H.R., Ciardi, B., Corstanje, A., Gasperin, F.d., Geus, E.d., Deller, A., Duscha, S., Eislöffel, J., Engels, D., Falcke, H., Fender, R., Ferrari, C., Frieswijk, W., Garrett, M.A., Grießmeier, J., Gunst, A.W., van Haarlem, M., Hassall, T.E., Heald, G., Hessels, J.W.T., Hoeft, M., Hörandel, J., Horneffer, A., Juette, E., Karastergiou, A., Klijn, W.F.A., Kondratiev, V.I., Kramer, M., Kuniyoshi, M., Kuper, G., Maat, P., Markoff, S., McFadden, R., McKay-Bukowski, D., McKean, J.P., Mulcahy, D.D., Munk, H., Nelles, A., Norden, M.J., Orru, E., Paas, H., Pandey-Pommier, M., Pandey, V.N., Pizzo, R., Polatidis, A.G., Rafferty, D., Reich, W., Röttgering, H., Scaife, A.M.M., Schwarz, D.J., Serylak, M., Sluman, J., Smirnov, O., Stappers, B.W., Tagger, M., Tang, Y., Tasse, C., ter Veen, S., Thoudam, S., Toribio, M.C., Vermeulen, R., van Weeren, R.J., Wise, M.W., Wucknitz, O., Yatawatta, 
S., Zarka, P., Zensus, J.A.: 2018, Tracking of an electron beam through the solar corona with LOFAR. Astron. Astrophys. 611, A57. DOI. ADS.

Masson, S., McCauley, P., Golub, L., Reeves, K.K., DeLuca, E.E.: 2014, Dynamics of the Transition Corona. Astrophys. J. 787, 145. DOI. ADS.

McCauley, P.I., Cairns, I.H., Morgan, J., Gibson, S.E., Harding, J.C., Lonsdale, C., Oberoi, D.: 2017, Type III Solar Radio Burst Source Region Splitting due to a Quasi-separatrix Layer. Astrophys. J. 851, 151. DOI. ADS.

Meléndez, J.L., Sawant, H.S., Fernandes, F.C.R., Benz, A.O.: 1999, Statistical analysis of high-frequency decimetric type III bursts. Solar Phys. 187, 77. DOI. ADS.

Melrose, D.B.: 2009, Coherent emission. In: Gopalswamy, N., Webb, D.F. (eds.) Universal Heliophysical Processes, IAU Symposium 257, 305. DOI. ADS.

Melrose, D.B., Dulk, G.A.: 1988, Implications of Liouville's theorem on the apparent brightness temperatures of solar radio bursts. Solar Phys. 116, 141. DOI. ADS.

Mercier, C., Chambe, G.: 2009, High Dynamic Range Images of the Solar Corona Between 150 and 450 MHz. Astrophys. J. Lett. 700, L137. DOI. ADS.

Mercier, C., Chambe, G.: 2015, Electron density and temperature in the solar corona from multifrequency radio imaging. Astron. Astrophys. 583, A101. DOI. ADS.

Mercier, C., Rosenberg, H.: 1974, Type III solar radio bursts observed at $169 \mathrm{MHz}$ - Height and relative positions in pairs. Solar Phys. 39, 193. DOI. ADS.

Mikić, Z., Linker, J.A., Schnack, D.D., Lionello, R., Tarditi, A.: 1999, Magnetohydrodynamic modeling of the global solar corona. Physics of Plasmas 6, 2217. DOI. ADS.

Mohan, A., Oberoi, D.: 2017, 4D Data Cubes from Radio-Interferometric Spectroscopic Snapshot Imaging. Solar Phys. 292, 168. DOI. ADS.

Morimoto, M.: 1964, The Height and Scatter of Sources of Type III Bursts of Solar Radio Emission. Pub. Astron. Soc. Japan 16, 163. ADS.

Morosan, D.E., Gallagher, P.T., Zucca, P., Fallows, R., Carley, E.P., Mann, G., Bisi, M.M., Kerdraon, A., Konovalenko, A.A., MacKinnon, A.L., Rucker, H.O., Thidé, B., Magdalenić, J., Vocks, C., Reid, H., Anderson, J., Asgekar, A., Avruch, I.M., Bentum, M.J., Bernardi, G., Best, P., Bonafede, A., Bregman, J., Breitling, F., Broderick, J., Brüggen, M., Butcher, H.R., Ciardi, B., Conway, J.E., de Gasperin, F., de Geus, E., Deller, A., Duscha, S., Eislöffel, J., Engels, D., Falcke, H., Ferrari, C., Frieswijk, W., Garrett, M.A., Grießmeier, J., Gunst, A.W., Hassall, T.E., Hessels, J.W.T., Hoeft, M., Hörandel, J., Horneffer, A., Iacobelli, M., Juette, E., Karastergiou, A., Kondratiev, V.I., Kramer, M., Kuniyoshi, M., Kuper, G., Maat, P., Markoff, S., McKean, J.P., Mulcahy, D.D., Munk, H., Nelles, A., Norden, M.J., Orru, E., Paas, H., Pandey-Pommier, M., Pandey, V.N., Pietka, G., Pizzo, R., Polatidis, A.G., Reich, W., Röttgering, H., Scaife, A.M.M., Schwarz, D., Serylak, M., Smirnov, O., Stappers, B.W., Stewart, A., Tagger, M., Tang, Y., Tasse, C., Thoudam, S., Toribio, C., Vermeulen, R., van Weeren, R.J., Wucknitz, O., Yatawatta, S., Zarka, P.: 2014, LOFAR tied-array imaging of Type III solar radio bursts. Astron. Astrophys. 568, A67. DOI. ADS.

Mugundhan, V., Ramesh, R., Kathiravan, C., Gireesh, G.V.S., Kumari, A., Hariharan, K., Barve, I.V.: 2018, The First Low-frequency Radio Observations of the Solar Corona on $\approx 200 \mathrm{~km}$ Long Interferometer Baseline. Astrophys. J. Lett. 855, L8. DOI. ADS.

Müller, D., Nicula, B., Felix, S., Verstringe, F., Bourgoignie, B., Csillaghy, A., Berghmans, D., Jiggens, P., García-Ortiz, J.P., Ireland, J., Zahniy, S., Fleck, B.: 2017, JHelioviewer. Time-dependent 3D visualisation of solar and heliospheric data. Astron. Astrophys. 606, A10. DOI. ADS.

Newkirk, G. Jr.: 1961, The Solar Corona in Active Regions and the Thermal Origin of the Slowly Varying Component of Solar Radio Radiation. Astrophys. J. 133, 983. DOI. ADS.

Offringa, A.R., van de Gronde, J.J., Roerdink, J.B.T.M.: 2012, A morphological algorithm for improving radio-frequency interference detection. Astron. Astrophys. 539, A95. DOI. ADS.

Offringa, A.R., McKinley, B., Hurley-Walker, N., Briggs, F.H., Wayth, R.B., Kaplan, D.L., Bell, M.E., Feng, L., Neben, A.R., Hughes, J.D., Rhee, J., Murphy, T., Bhat, N.D.R., Bernardi, G., Bowman, J.D., Cappallo, R.J., Corey, B.E., Deshpande, A.A., Emrich, D., Ewall-Wice, A., Gaensler, B.M., Goeke, R., Greenhill, L.J., Hazelton, B.J., Hindson, L., Johnston-Hollitt, M., Jacobs, D.C., Kasper, 
J.C., Kratzenberg, E., Lenc, E., Lonsdale, C.J., Lynch, M.J., McWhirter, S.R., Mitchell, D.A., Morales, M.F., Morgan, E., Kudryavtseva, N., Oberoi, D., Ord, S.M., Pindor, B., Procopio, P., Prabu, T., Riding, J., Roshi, D.A., Shankar, N.U., Srivani, K.S., Subrahmanyan, R., Tingay, S.J., Waterson, M., Webster, R.L., Whitney, A.R., Williams, A., Williams, C.L.: 2014, WSCLEAN: an implementation of a fast, generic wide-field imager for radio astronomy. Mon. Not. Roy. Astron. Soc. 444, 606. DOI. ADS.

Offringa, A.R., Wayth, R.B., Hurley-Walker, N., Kaplan, D.L., Barry, N., Beardsley, A.P., Bell, M.E., Bernardi, G., Bowman, J.D., Briggs, F., Callingham, J.R., Cappallo, R.J., Carroll, P., Deshpande, A.A., Dillon, J.S., Dwarakanath, K.S., Ewall-Wice, A., Feng, L., For, B.-Q., Gaensler, B.M., Greenhill, L.J., Hancock, P., Hazelton, B.J., Hewitt, J.N., Hindson, L., Jacobs, D.C., Johnston-Hollitt, M., Kapińska, A.D., Kim, H.-S., Kittiwisit, P., Lenc, E., Line, J., Loeb, A., Lonsdale, C.J., McKinley, B., McWhirter, S.R., Mitchell, D.A., Morales, M.F., Morgan, E., Morgan, J., Neben, A.R., Oberoi, D., Ord, S.M., Paul, S., Pindor, B., Pober, J.C., Prabu, T., Procopio, P., Riding, J., Udaya Shankar, N., Sethi, S., Srivani, K.S., Staveley-Smith, L., Subrahmanyan, R., Sullivan, I.S., Tegmark, M., Thyagarajan, N., Tingay, S.J., Trott, C.M., Webster, R.L., Williams, A., Williams, C.L., Wu, C., Wyithe, J.S., Zheng, Q.: 2015, The Low-Frequency Environment of the Murchison Widefield Array: Radio-Frequency Interference Analysis and Mitigation. PASA 32, e008. DOI. ADS.

Ord, S.M., Crosse, B., Emrich, D., Pallot, D., Wayth, R.B., Clark, M.A., Tremblay, S.E., Arcus, W., Barnes, D., Bell, M., Bernardi, G., Bhat, N.D.R., Bowman, J.D., Briggs, F., Bunton, J.D., Cappallo, R.J., Corey, B.E., Deshpande, A.A., deSouza, L., Ewell-Wice, A., Feng, L., Goeke, R., Greenhill, L.J., Hazelton, B.J., Herne, D., Hewitt, J.N., Hindson, L., Hurley-Walker, N., Jacobs, D., Johnston-Hollitt, M., Kaplan, D.L., Kasper, J.C., Kincaid, B.B., Koenig, R., Kratzenberg, E., Kudryavtseva, N., Lenc, E., Lonsdale, C.J., Lynch, M.J., McKinley, B., McWhirter, S.R., Mitchell, D.A., Morales, M.F., Morgan, E., Oberoi, D., Offringa, A., Pathikulangara, J., Pindor, B., Prabu, T., Procopio, P., Remillard, R.A., Riding, J., Rogers, A.E.E., Roshi, A., Salah, J.E., Sault, R.J., Udaya Shankar, N., Srivani, K.S., Stevens, J., Subrahmanyan, R., Tingay, S.J., Waterson, M., Webster, R.L., Whitney, A.R., Williams, A., Williams, C.L., Wyithe, J.S.B.: 2015, The Murchison Widefield Array Correlator. PASA 32, e006. DOI. ADS.

Parenti, S., Bromage, B.J.I., Poletto, G., Noci, G., Raymond, J.C., Bromage, G.E.: 2000, Characteristics of solar coronal streamers. Element abundance, temperature and density from coordinated CDS and UVCS SOHO observations. Astron. Astrophys. 363, 800. ADS.

Pesnell, W.D., Thompson, B.J., Chamberlin, P.C.: 2012, The Solar Dynamics Observatory (SDO). Solar Phys. 275, 3. DOI. ADS.

Pick, M., Kerdraon, A., Auchère, F., Stenborg, G., Bouteille, A., Soubrié, E.: 2009, Coronal and Interplanetary Structures Associated with Type III Bursts. Solar Phys. 256, 101. DOI. ADS.

Poquerusse, M.: 1994, Relativistic type 3 solar radio bursts. Astron. Astrophys. 286, 611. ADS.

Poquerusse, M., Steinberg, J.L., Caroubalos, C., Dulk, G.A., MacQueen, R.M.: 1988, Measurement of the 3-dimensional positions of type III bursts in the solar corona. Astron. Astrophys. 192, 323. ADS.

Prestage, N.P., Luckhurst, R.G., Paterson, B.R., Bevins, C.S., Yuile, C.G.: 1994, A new radiospectrograph at Culgoora. Solar Phys. 150, 393. DOI. ADS.

Pucci, S., Lie-Svendsen, Ø., Esser, R.: 2010, Elemental Abundances in the Fast Solar Wind Emanating from Chromospheric Funnels. Astrophys. J. 709, 993. DOI. ADS.

Ramesh, R., Nataraj, H.S., Kathiravan, C., Sastry, C.V.: 2006, The Equatorial Background Solar Corona during Solar Minimum. Astrophys. J. 648, 707. DOI. ADS.

Raymond, J.C., McCauley, P.I., Cranmer, S.R., Downs, C.: 2014, The Solar Corona as Probed by Comet Lovejoy (C/2011 W3). Astrophys. J. 788, 152. DOI. ADS.

Reid, H.A.S., Ratcliffe, H.: 2014, A review of solar type III radio bursts. Research in Astronomy and Astrophysics 14, 773. DOI. ADS.

Reid, H.A.S., Vilmer, N., Kontar, E.P.: 2014, The low-high-low trend of type III radio burst starting frequencies and solar flare hard X-rays. Astron. Astrophys. 567, A85. DOI. ADS.

Reid, M.J., Schneps, M.H., Moran, J.M., Gwinn, C.R., Genzel, R., Downes, D., Roennaeng, B.: 1988, The distance to the center of the Galaxy - H2O maser proper motions in Sagittarius B2(N). Astrophys. J. 330, 809. DOI. ADS.

Riddle, A.C.: 1974, On the Observation of Scattered Radio Emission from Sources in the Solar Corona. 
Solar Phys. 35, 153. DOI. ADS.

Robinson, P.A., Cairns, I.H.: 1998, Fundamental and Harmonic Emission in Type III Solar Radio Bursts - I. Emission at a Single Location or Frequency. Solar Phys. 181, 363. DOI. ADS.

Robinson, P.A., Cairns, I.H.: 2000, Theory of Type III And Type II Solar Radio Emissions. Washington DC American Geophysical Union Geophysical Monograph Series 119, 37. DOI. ADS.

Robinson, R.D.: 1983, Scattering of radio waves in the solar corona. Proceedings of the Astronomical Society of Australia 5, 208. DOI. ADS.

Saito, K., Owaki, N.: 1967, An Active Region Streamer of the Solar Corona. Pub. Astron. Soc. Japan 19, 535. ADS.

Saito, K., Poland, A.I., Munro, R.H.: 1977, A study of the background corona near solar minimum. Solar Phys. 55, 121. DOI. ADS.

Sastry, C.V.: 1994, Observations of the continuum radio emission from the undisturbed Sun at a wavelength of 8.7 meters. Solar Phys. 150, 285. DOI. ADS.

Schrijver, C.J., Sandman, A.W., Aschwanden, M.J., De Rosa, M.L.: 2004, The Coronal Heating Mechanism as Identified by Full-Sun Visualizations. Astrophys. J. 615, 512. DOI. ADS.

Spadaro, D., Susino, R., Ventura, R., Vourlidas, A., Landi, E.: 2007, Physical parameters of a mid-latitude streamer during the declining phase of the solar cycle. Astron. Astrophys. 475, 707. DOI. ADS.

Steinberg, J.L., Hoang, S., Dulk, G.A.: 1985, Evidence of scattering effects on the sizes of interplanetary Type III radio bursts. Astron. Astrophys. 150, 205. ADS.

Steinberg, J.L., Hoang, S., Lecacheux, A., Aubier, M.G., Dulk, G.A.: 1984, Type III radio bursts in the interplanetary medium - The role of propagation. Astron. Astrophys. 140, 39. ADS.

Stewart, R.T.: 1972, Relative Positions of Fundamental and Second Harmonic Type III Bursts. Proceedings of the Astronomical Society of Australia 2, 100. DOI. ADS.

Stewart, R.T.: 1974, Harmonic ratios of inverted-U type III bursts. Solar Phys. 39, 451. DOI. ADS.

Stewart, R.T.: 1976, Source heights of metre wavelength bursts of spectral types I and III. Solar Phys. 50, 437. DOI. ADS.

Subramanian, K.R.: 2004, Brightness temperature and size of the quiet Sun at $34.5 \mathrm{MHz}$. Astron. Astrophys. 426, 329. DOI. ADS.

Sutinjo, A., O’Sullivan, J., Lenc, E., Wayth, R.B., Padhi, S., Hall, P., Tingay, S.J.: 2015, Understanding instrumental Stokes leakage in Murchison Widefield Array polarimetry. Radio Science 50, 52. DOI. ADS.

Thejappa, G., Kundu, M.R.: 1992, Unusually low coronal radio emission at the solar minimum. Solar Phys. 140, 19. DOI. ADS.

Thejappa, G., MacDowall, R.J.: 2008, Effects of Scattering on Radio Emission from the Quiet Sun at Low Frequencies. Astrophys. J. 676, 1338. DOI. ADS.

Tingay, S.J., Goeke, R., Bowman, J.D., Emrich, D., Ord, S.M., Mitchell, D.A., Morales, M.F., Booler, T., Crosse, B., Wayth, R.B., Lonsdale, C.J., Tremblay, S., Pallot, D., Colegate, T., Wicenec, A., Kudryavtseva, N., Arcus, W., Barnes, D., Bernardi, G., Briggs, F., Burns, S., Bunton, J.D., Cappallo, R.J., Corey, B.E., Deshpande, A., Desouza, L., Gaensler, B.M., Greenhill, L.J., Hall, P.J., Hazelton, B.J., Herne, D., Hewitt, J.N., Johnston-Hollitt, M., Kaplan, D.L., Kasper, J.C., Kincaid, B.B., Koenig, R., Kratzenberg, E., Lynch, M.J., Mckinley, B., Mcwhirter, S.R., Morgan, E., Oberoi, D., Pathikulangara, J., Prabu, T., Remillard, R.A., Rogers, A.E.E., Roshi, A., Salah, J.E., Sault, R.J., Udaya-Shankar, N., Schlagenhaufer, F., Srivani, K.S., Stevens, J., Subrahmanyan, R., Waterson, M., Webster, R.L., Whitney, A.R., Williams, A., Williams, C.L., Wyithe, J.S.B.: 2013, The Murchison Widefield Array: The Square Kilometre Array Precursor at Low Radio Frequencies. PASA 30, e007. DOI. ADS.

Trottet, G., Pick, M., House, L., Illing, R., Sawyer, C., Wagner, W.: 1982, An association between coronal structures and type III burst sources. Astron. Astrophys. 111, 306. ADS.

Wang, T., Reginald, N.L., Davila, J.M., St. Cyr, O.C., Thompson, W.T.: 2017, Variation in Coronal Activity from Solar Cycle 24 Minimum to Maximum Using Three-Dimensional Reconstructions of the Coronal Electron Density from STEREO/COR1. Solar Phys. 292, 97. DOI. ADS.

Wild, J.P., McCready, L.L.: 1950, Observations of the Spectrum of High-Intensity Solar Radiation at Metre Wavelengths. I. The Apparatus and Spectral Types of Solar Burst Observed. Australian Journal 


\section{of Scientific Research A Physical Sciences 3, 387. DOI. ADS.}

Wild, J.P., Sheridan, K.V., Neylan, A.A.: 1959, An Investigation of the Speed of the Solar Disturbances responsible for Type III Radio Bursts. Australian Journal of Physics 12, 369. DOI. ADS.

Woo, R.: 2007, Filamentary Structures of Coronal White-Light Images. Solar Phys. 241, 251. DOI. ADS. 